\title{
PLANES, BRANES AND AUTOMORPHISMS I. STATIC BRANES
}

\author{
BS ACHARYA, JM FIGUEROA-O'FARRILL, AND B SPENCE
}

\begin{abstract}
This is the first of a series of papers devoted to the group-theoretical analysis of the conditions which must be satisfied for a configuration of intersecting M5-branes to be supersymmetric. In this paper we treat the case of static branes. We start by associating (a maximal torus of) a different subgroup of $\operatorname{Spin}_{10}$ with each of the equivalence classes of supersymmetric configurations of two M5-branes at angles found by Ohta \& Townsend. We then consider configurations of more than two intersecting branes. Such a configuration will be supersymmetric if and only if the branes are $G$-related, where $G$ is a subgroup of $\operatorname{Spin}_{10}$ contained in the isotropy of a spinor. For each such group we determine (a lower bound for) the fraction of the supersymmetry which is preserved. We give examples of configurations consisting of an arbitrary number of non-coincident intersecting fivebranes with fractions: $\frac{1}{32}, \frac{1}{16}$, $\frac{3}{32}, \frac{1}{8}, \frac{5}{32}, \frac{3}{16}$, and $\frac{1}{4}$, and we determine the resulting (calibrated) geometry.
\end{abstract}

\section{Contents}

1. Introduction 2

2. Supersymmetric pairs of M5-branes at angles 3

\begin{tabular}{lll}
\hline 3. & Group-theoretical analysis & 7
\end{tabular}

\begin{tabular}{lll}
\hline 3.1. & The results of Ohta \& Townsend & 7
\end{tabular}

3.2 . Some regular subgroups of Spin $_{10}$

$\begin{array}{lll}3.3 . & \text { Detailed analysis } & 10\end{array}$

\begin{tabular}{lll}
\hline 3.4. & Pointlike intersections & 10
\end{tabular}

$\begin{array}{lll}3.5 . & \text { Stringlike intersections } & 12\end{array}$

$\begin{array}{lll}3.6 . & \text { Two-dimensional intersections } & 14\end{array}$

\begin{tabular}{|lll}
\hline 3.7. & Three-dimensional intersections & 15
\end{tabular}

$\begin{array}{lll}3.8 . & \text { Summary of results } & 16\end{array}$

\begin{tabular}{lll}
\hline $4 . \quad$ Multiple intersections & 17
\end{tabular}

$\begin{array}{lll}4.1 . & \text { Statement of the problem } & 17\end{array}$

4.2. Supersymmetric configurations of $G$-related planes 18

4.3. Geometry of intersecting brane configurations 22

\begin{tabular}{|ll}
\hline $5 . \quad$ Conclusions and open problems & 24
\end{tabular}

Acknowledgements 25

\begin{tabular}{ll}
\hline References & 25
\end{tabular}

Date: June 19, 2021. 


\section{IntRoduCtion}

The complete classification of supersymmetric solutions of elevendimensional supergravity would be an important step towards understanding the true nature of M-theory; but this seems to be a very difficult problem. A more manageable task seems to be the classification of supersymmetric configurations which locally look like intersecting M-branes. Earlier works on this topic [23, 13, 26, 5] considered branes intersecting orthogonally. These configurations always preserve a fraction $\frac{1}{2^{n}}$ of the supersymmetry, and all such fractions occur: $\frac{1}{4}$, $\frac{1}{8}, \frac{1}{16}$, and $\frac{1}{32}$. The possibility of more general-i.e., non-orthogonalintersections was originally considered in [6] who noticed that branes related by $\mathrm{SU}_{n} \subset \mathrm{SO}_{2 n}$ transformations still preserve some supersymmetry. In [12] configurations in which the branes are related by $\mathrm{Sp}_{2} \subset \mathrm{SO}_{8}$ transformations were shown to be dual to Kaluza-Klein supergravity on eight-dimensional hyperkähler manifolds. Also in [7, 4, 9, 3] some of the configurations of branes at angles were shown to be dual to branes which intersect orthogonally. Of course, this is impossible for configurations preserving a fraction which is not a power of 2. Such configurations were first discussed by Townsend [25], who initiated the classification of the supersymmetric configurations of a pair of static M5-branes at angles, a classification completed with Ohta in 22]; although see also [19] for some earlier but incomplete results. In a previous paper [1] we interpreted the results of [22] in terms of calibrated geometry and extended them to an arbitrary number of M5-branes. Similar results in a somewhat different context have been obtained in [15, 14] also using techniques of calibrated geometry.

The purpose of the present series of papers is to establish a grouptheoretical framework in which to phrase the analysis of these conditions and in which to study the multiple intersection problem. In this first paper we will consider the case of static branes, as in the work of Ohta \& Townsend. In a forthcoming paper [2], hereafter referred to as Part II, we will treat the general case. We will assign a different subgroup $G$ of $\operatorname{Spin}_{10}$ to each of the configurations in [22], in such a way that the preserved supersymmetry corresponds to the spinors left invariant by $G$, or in the case of two branes, by its maximal torus. Many of the groups which occur are possible holonomy groups of spin riemannian manifolds and, by construction, all of them leave invariant a nonzero number of spinors. This lends further evidence to the comments in 22 concerning the possible duality between these configurations of intersecting branes and Kaluza-Klein reduction of eleven-dimensional supergravity on manifolds of reduced holonomy [12]. While we do not consider the exact brane solutions in this paper nor discuss their Kaluza-Klein duals, we hope to return to this question in a future publication. 
This paper is organised as follows. In the next section we recast the analysis of Ohta \& Townsend in an intrinsically group-theoretical fashion (see also [1]). This will facilitate the ensuing discussion. In Section 3 we perform the detailed group-theoretical analysis and associate a different group with each class of configurations. In Section 4 we study the case of supersymmetric configurations involving more than two intersecting branes. We show that any such configuration consists of branes which are "G-related" (see below for a precise definition), where $G \subset \operatorname{Spin}_{10}$ leaves a nonzero number of spinors invariant, and given any such $G$ we determine (a lower bound for) the fraction of the supersymmetry that a configuration of $G$-related branes will preserve. This yields examples of intersecting brane configurations involving an arbitrary number of non-coincident branes which preserve the following possible fractions of the supersymmetry: $\frac{1}{32}, \frac{1}{16}, \frac{3}{32}, \frac{1}{8}, \frac{5}{32}, \frac{3}{16}, \frac{1}{4}$ and $\frac{1}{2}$. We also comment on the geometry of some of these intersecting brane configurations. Finally, section 5 summarises some of the open problems related to this work.

\section{Supersymmetric Pairs of M5-Branes AT ANGLES}

In this section we set up our notation and review the approach of Ohta \& Townsend [22]. A less detailed version of this analysis has appeared in [1]. Let us consider the M5-brane solution. Let $\left(x^{\mu}\right)$ denote the eleven-dimensional coordinates, where $\left(x^{0}, x^{1}, \ldots, x^{5}\right)$ are coordinates along the brane and $\left(x^{6}, \ldots, x^{9}, x^{\natural}\right)$ are coordinates transverse to the brane. Far away from the brane, the metric is asymptotically flat, so that the Killing spinors of the supergravity solution have constant asymptotic values $\varepsilon$, obeying

$$
\Gamma_{012345} \varepsilon=\varepsilon,
$$

where $\varepsilon$ is a real 32-component spinor of $\operatorname{Spin}_{10,1}$. We think of $\operatorname{Spin}_{10,1}$ as contained in the Clifford algebra $\mathrm{C} \ell_{1,10}$ generated by the $\Gamma_{M}$. Provided we only deal with one brane, it is possible to choose coordinates so that the brane is stretched along these directions; but the moment we have to consider two or more branes, particularly if they intersect nonorthogonally, this notation becomes cumbersome, since not all branes can be described so conveniently. Moreover our aim in this paper is not to analyse the global properties of branes, but their local properties at the point of intersection. In fact, we could be analysing singularities in a single brane which is immersed (rather than embedded) in the spacetime. We will therefore recast the work of [22] in terms of tangent planes at a point to the branes themselves.

Let us fix a point $x$ in the spacetime $M$ and an orthonormal frame $e_{0}, e_{1}, \ldots, e_{9}, e_{\natural}$ for the tangent space at $x$. This allows us to identify the tangent space $T_{x} M$ with eleven-dimensional Minkowski spacetime 
$\mathbb{M}^{10,1}$. We will further decompose $\mathbb{M}^{10,1}=\mathbb{R} e_{0} \oplus \mathbb{E}^{10}$. This decomposition is preserved by an $\mathrm{SO}_{10}$ subgroup of $\mathrm{SO}_{10,1}$. As in 22 we will restrict ourselves to configurations for which the tangent plane to the worldvolume of a given M5-brane passing through $x$ is spanned by $e_{0}, v_{1}, \ldots, v_{5}$, where $v_{i}$ are orthonormal vectors in $\mathbb{E}^{10}$. In particular all these planes share a common timelike direction, whence they are static relative to one another. We will lift this restriction in Part II. Suppose moreover that the brane is given the orientation defined by $e_{0} \wedge v_{1} \wedge \cdots \wedge v_{5}$. We will therefore be able to associate with each such brane at $x$ a 5 -vector $\xi=v_{1} \wedge \cdots \wedge v_{5}$ in $\wedge^{5} \mathbb{E}^{10}$. Conversely, to any given unit simple 5 -vector $\xi=v_{1} \wedge \cdots \wedge v_{5}$, we associate an oriented 5 -plane given by the span of the $v_{i}$. The condition for supersymmetry (11) can be rewritten more generally as

$$
\left(e_{0} \wedge \xi\right) \cdot \varepsilon=\varepsilon,
$$

where $\cdot$ stands for Clifford multiplication and where we have used implicitly the isomorphism of the Clifford algebra $\mathrm{C} \ell_{1,10}$ with the exterior algebra $\bigwedge M^{10,1}$. When $\xi=e_{1} \wedge e_{2} \wedge \cdots \wedge e_{5}$, equation (2) agrees with equation (11).

Now suppose that we are given two M5-branes through $x$ with tangent planes $\xi$ and $\eta$. This configuration will be supersymmetric if there exists a nonzero spinor $\varepsilon$ for which

$$
\left(e_{0} \wedge \xi\right) \cdot \varepsilon=\varepsilon \quad \text { and } \quad\left(e_{0} \wedge \eta\right) \cdot \varepsilon=\varepsilon .
$$

Because $\mathrm{SO}_{10}$ acts transitively on the space of 5-planes, there exists a rotation $R$ in $\mathrm{SO}_{10}$ which transforms $\xi$ to $\eta$. Because $R$ is conjugate to any given maximal torus of $\mathrm{SO}_{10}$, there exists a choice of orthonormal frame $e_{i}$ for which $\xi=e_{1} \wedge e_{3} \wedge \cdots \wedge e_{9}$ and

$$
\eta=R(\theta) \xi=\left(\cos \theta_{1} e_{1}+\sin \theta_{1} e_{2}\right) \wedge \cdots \wedge\left(\cos \theta_{5} e_{9}+\sin \theta_{5} e_{\natural}\right),
$$

where $R(\theta)$ is the block-diagonal matrix

$$
R(\theta)=\left(\begin{array}{lllll}
R_{12}\left(\theta_{1}\right) & & & & \\
& R_{34}\left(\theta_{2}\right) & & & \\
& & R_{56}\left(\theta_{3}\right) & & \\
& & & R_{78}\left(\theta_{4}\right) & \\
& & & & R_{9 \natural}\left(\theta_{5}\right)
\end{array}\right),
$$

each $R_{j k}(\vartheta)$ being the rotation by an angle $\vartheta$ in the 2-plane spanned by $e_{j}$ and $e_{k}$. The angles $\left(\theta_{i}\right)$ are of course not unique, because having conjugated $R$ into a given maximal torus, we can still act with Weyl transformations.

The Weyl group $\mathfrak{W}$ of $\mathrm{SO}_{10}$ is described as follows. Consider the group of permutations $\sigma$ of the ten-element set $\{-5, \ldots,-1,1, \ldots, 5\}$ such that $\sigma(-j)=-\sigma(j)$. This group is isomorphic to the semidirect product $\mathfrak{S}_{5} \ltimes\left(\mathbb{Z}_{2}\right)^{5}$, where the symmetric group $\mathfrak{S}_{5}$ acts on $\left(\mathbb{Z}_{2}\right)^{5}$ interchanging the factors. The Weyl group of $\mathrm{SO}_{10}$ is then the subgroup of 
index 2 consisting of even permutations. It has order 1920. Its action on the maximal torus is given by $\left(\theta_{1}, \ldots, \theta_{5}\right) \mapsto\left(\theta_{\sigma(1)}, \ldots, \theta_{\sigma(5)}\right)$ with the convention that $\theta_{-j}=-\theta_{j}$.

Let $\mathcal{M}$ denote the space of relative configurations of two 5-planes in $\mathbb{E}^{10}$; that is, $\mathcal{M} \cong \mathbb{T} / \mathfrak{W}$ is the quotient of the maximal torus $\mathbb{T}$ of $\mathrm{SO}_{10}$ by the action of the Weyl group. If $\theta \equiv\left(\theta_{i}\right)$ are any five angles, we will let $[\theta] \in \mathcal{M}$ denote their equivalence class under the action of the Weyl group. The subset $\mathcal{M}_{\text {susy }} \subset \mathcal{M}$ consists of those angles $[\theta]$ for which the intersecting brane configuration defined by $\xi$ and $R(\theta) \xi$ preserves some supersymmetry. In other words, $\mathcal{M}_{\text {susy }}$ is the subset of $\mathcal{M}$ for which there is at least one nonzero spinor $\varepsilon$ which solves the following equations:

$$
\left(e_{0} \wedge \xi\right) \cdot \varepsilon=\varepsilon \quad \text { and } \quad\left(e_{0} \wedge R(\theta) \xi\right) \cdot \varepsilon=\varepsilon .
$$

For each point $[\theta]$ in $\mathcal{M}$, let $32 \nu([\theta])$ be equal to the number of linearly independent solutions $\varepsilon$ to (4). Therefore $\nu$ defines a (discontinuous) function on $\mathcal{M}$ which can be interpreted as the fraction of the supersymmetry preserved by the configuration. A priori $\nu$ can take any of the values $0, \frac{1}{32}, \frac{1}{16}, \frac{3}{32}, \ldots, \frac{1}{2}$, but as we will see not all values actually occur. In particular, $\nu([\theta])=\frac{1}{2}$ if and only if all the angles vanish, whereas $\nu([\theta]) \neq 0$ if and only if $[\theta]$ belongs to $\mathcal{M}_{\text {susy }}$.

Let $\widehat{R}$ denote any one of the two possible lifts to $\operatorname{Spin}_{10}$ of the $\mathrm{SO}_{10}$ rotation $R$. Then the second equation in (4) can be written as follows:

$$
\widehat{R}(\theta) \cdot\left(e_{0} \wedge \xi\right) \cdot \widehat{R}(\theta)^{-1} \cdot \varepsilon=\varepsilon .
$$

Using the fact that

$$
\left(e_{0} \wedge \xi\right) \cdot \widehat{R}(\theta)^{-1}=\widehat{R}(\theta) \cdot\left(e_{0} \wedge \xi\right)
$$

together with the first equation in (4), we arrive at

$$
\widehat{R}(\theta)^{2} \cdot \varepsilon=\varepsilon,
$$

with the same equation resulting for the other possible lift $-\widehat{R}(\theta)$.

$\operatorname{Spin}_{10}$ has two complex half-spin representations $\Delta_{ \pm}$, obeying $\Delta_{+}^{*} \cong$ $\Delta_{-}$. Therefore their direct sum $\Delta_{+} \oplus \Delta_{-}$has a real structure. The underlying real representation $\Delta$, defined by $\Delta \otimes_{\mathbb{R}} \mathbb{C}=\Delta_{+} \oplus \Delta_{-}$, is the real spinor representation of $\operatorname{Spin}_{10,1}$ to which $\varepsilon$ belongs, whence we can think of $\varepsilon$ as a conjugate pair of spinors, $\varepsilon=\left(\psi, \psi^{*}\right) \in \Delta_{+} \oplus \Delta_{-}$. In this way, equation (6) simply becomes the statement that $\psi \in \Delta_{+}$ is invariant under the action of $\widehat{R}(\theta)^{2} \in \operatorname{Spin}_{10}$. As we shall see in more detail below, the real and imaginary parts of each such $\psi$ give two real solutions of (6), but exactly one of each such pair also obeys the first equation in (4). Therefore the number of linearly independent solutions of (4) are in one-to-one correspondence with the number of positive-chirality spinors $\psi \in \Delta_{+}$of $\operatorname{Spin}_{10}$ which are left invariant by $\widehat{R}(\theta)^{2}$. 
Notice that $\widehat{R}(\theta)^{2}$ is given explicitly by

$$
\widehat{R}(\theta)^{2}=\left(\cos \theta_{1}-\sin \theta_{1} \Gamma_{12}\right) \cdots\left(\cos \theta_{5}-\sin \theta_{5} \Gamma_{9 \natural}\right) \in \mathrm{C} \ell_{1,10},
$$

which is an element in the maximal torus of $\operatorname{Spin}_{10}$ corresponding to the chosen maximal torus for $\mathrm{SO}_{10}$. The maximal torus of $\mathrm{Spin}_{10}$ acts diagonally on the space $\Delta_{+}$of positive-chirality spinors, with eigenvalues the exponentials of the weights. The highest weight vector of $\Delta_{ \pm}$ is given by $\frac{1}{2}(1,1,1,1, \pm 1)$. All other weights in $\Delta_{ \pm}$are Weyl-related to the highest weight: in particular they have multiplicity one. Notice also that $\lambda$ is a weight of $\Delta_{+}$if and only if $-\lambda$ is a weight of $\Delta_{-}$. Now let $\lambda$ be a weight of $\Delta_{+}$and let $\varepsilon_{\lambda} \in \Delta_{+}$denote the unique (up to scale) weight vector of weight $\lambda$. Let $\varepsilon_{-\lambda}=\varepsilon_{\lambda}^{*}$ denote the corresponding weight vector in $\Delta_{-}$. Taken together, $\varepsilon_{ \pm \lambda}$ are a complex basis for $\Delta_{+} \oplus \Delta_{-}$. If $\lambda=\frac{1}{2}\left(\sigma_{1}, \sigma_{2}, \ldots, \sigma_{5}\right)$, where the $\sigma_{i}$ are signs such that their product is positive, then

$$
\widehat{R}(\theta)^{2} \cdot \varepsilon_{ \pm \lambda}=\exp \left( \pm \sqrt{-1} \sum_{i} \sigma_{i} \theta_{i}\right) \varepsilon_{ \pm \lambda}
$$

Therefore the spinors left invariant by $\widehat{R}(\theta)^{2}$ are in one-to-one correspondence with the weights $\lambda$ of $\Delta_{+}$for which

$$
\lambda \cdot \theta \equiv \sum_{i} \sigma_{i} \theta_{i}=0 \quad(\bmod 2 \pi) .
$$

Equivariance under the Weyl group guarantees that if we Weyl transform the angles $\theta$ we simply Weyl transform the solutions $\lambda$. In particular, the fraction $\nu([\theta])$, which is $\frac{1}{32} \times$ the number of weights $\lambda$ obeying $\lambda \cdot \theta=0(\bmod 2 \pi)$, is a well defined function on $\mathcal{M}$.

Already we can characterise the space of supersymmetric configurations $\mathcal{M}_{\text {susy }}$. Let $\theta$ be some angles satisfying equation (7) for some weight $\lambda$ of $\Delta_{+}$. This weight is in the Weyl orbit of the highest weight $\lambda_{\max }=\frac{1}{2}(1,1,1,1,1)$, hence by Weyl equivariance there will be some angles $\theta^{\prime}$, Weyl-related to $\theta$, for which $\lambda_{\max } \cdot \theta^{\prime}=\sum_{i} \theta_{i}^{\prime}=0 \bmod 2 \pi$. In other words, we arrive at the following elegant characterisation of $\mathcal{M}_{\text {susy }} 22$ :

$$
\mathcal{M}_{\text {susy }}=\left\{[\theta] \in \mathcal{M} \mid \sum_{i=1}^{5} \theta_{i}=0 \quad(\bmod 2 \pi)\right\} .
$$

For generic $[\theta] \in \mathcal{M}_{\text {susy }}$, there will be a unique weight $\lambda$ of $\Delta_{+}$which satisfies $\lambda \cdot \theta=0(\bmod 2 \pi)$. As we now explain this configuration preserves $\frac{1}{32}$ of the supersymmetry.

We shall find it convenient to first examine the action of $\widehat{R}(\theta)$ on $\Delta$. Let $e_{\lambda}=\varepsilon_{\lambda}+\varepsilon_{-\lambda}$ and $f_{\lambda}=\sqrt{-1}\left(\varepsilon_{\lambda}-\varepsilon_{-\lambda}\right)$ denote the real and imaginary parts of the complex weight vector $\varepsilon_{\lambda}$. The set $\left\{e_{\lambda}, f_{\lambda}\right\}$ as $\lambda$ runs over the weights of $\Delta_{+}$(or equivalently $\Delta_{-}$, since $e_{-\lambda}=e_{\lambda}$ and $f_{-\lambda}=-f_{\lambda}$ ), is a real basis for $\Delta$. In this basis, $\widehat{R}(\theta)^{2}$ is no longer 
diagonal, but block-diagonal with $2 \times 2$ blocks. On the two-dimensional subspace of $\Delta$ spanned by $e_{\lambda}$ and $f_{\lambda}$, it acts with matrix

$$
\left(\begin{array}{rr}
\cos \lambda \cdot \theta & \sin \lambda \cdot \theta \\
-\sin \lambda \cdot \theta & \cos \lambda \cdot \theta
\end{array}\right)
$$

whence if $\lambda$ satisfies (7), both $e_{\lambda}$ and $f_{\lambda}$ are left invariant. It might seem as if we had two solutions per weight, but in fact the first equation in (4) halves the number of solutions. To see this, notice that for our choice of 5 -plane $\xi=e_{1} \wedge e_{3} \wedge \cdots \wedge e_{9}$, this equation becomes

$$
\Gamma_{013579} \varepsilon=\varepsilon .
$$

Because $\widetilde{\Gamma} \equiv \Gamma_{013579}$ anticommutes with the Cartan generators $\Gamma_{12}$, $\Gamma_{34}, \Gamma_{56}, \Gamma_{78}$, and $\Gamma_{9 \natural}$, it preserves the subspace $\Delta_{0} \subset \Delta$ associated with the weights $\lambda$ obeying (17). Because $\widetilde{\Gamma}^{2}=+\mathbb{1}$, it decomposes $\Delta_{0}$ into $\Delta_{0}^{+} \oplus \Delta_{0}^{-}$according to its eigenvalue. Equation (9) says that $\varepsilon$ belongs to $\Delta_{0}^{+}$. To show that $\Delta_{0}^{ \pm}$have the same dimension, it suffices to show that $\Gamma_{0}$ relates them. Indeed, $\Gamma_{0}$ commutes with the Cartan generators, so that it preserves $\Delta_{0}$, and anticommutes with $\widetilde{\Gamma}$ so that it maps $\Delta_{0}^{+}$to $\Delta_{0}^{-}$isomorphically.

Therefore of each pair of solutions $e_{\lambda}, f_{\lambda}$ of (6), exactly one linear combination survives (9). For a generic point $[\theta] \in \mathcal{M}_{\text {susy }}$, there is exactly one weight $\lambda$ in $\Delta_{+}$which satisfies (7). Therefore generically there are two linearly independent solutions $e_{\lambda}$ and $f_{\lambda}$ of equation (6), one of which satisfies equation (9). In other words, the configuration with characterising angles $[\theta]$ preserves $\frac{1}{32}$ of the supersymmetry.

As described by Ohta \& Townsend [22] there are other configurations preserving a larger fraction $\nu$ of the supersymmetry. In the next section we examine the group theory behind these special configurations. In particular, we will be able to assign a different subgroup of $\operatorname{Spin}_{10}$ to each such configuration. This "automorphism" group of the brane configuration often coincides with the holonomy group of a riemannian spin manifolds possessing parallel spinors.

\section{Group-Theoretical ANALYSis}

In this section we will show how the different supersymmetric configurations in 22 correspond to different subgroups of $\operatorname{Spin}_{10}$ leaving some spinor(s) invariant; but before getting into the group-theoretical description let us summarise the results of [22].

3.1. The results of Ohta \& Townsend. In solving equations (4), or equivalently the first equation in (4) and equation (6), it is convenient to label the solutions according to two parameters: the fraction $\nu$ of the supersymmetry that the configuration preserves, and the codimension $d$ of the intersection of the two fivebranes relative to any one of the fivebranes. A configuration of two coincident branes have a fivedimensional intersection, whence its codimension is zero. At the other 
extreme, a configuration of two fivebranes which only intersect in a point has codimension 5 . In most cases, the codimension will agree with the number of nonzero angles in the rotation matrix $R(\theta)$. Discrepancy can occur only if any of the angles are equal to $\pm \pi$, in which case the planes are antiparallel and hence coincide up to orientation. In terms of these labels, the solutions found in [22] are summarised in Table 11. Missing from the table are a configuration with $d=5$ and $\nu=\frac{5}{32}$ and one with $d=4$ and $\nu=\frac{1}{4}$. These configurations are associated with finite subgroups of $\operatorname{Spin}_{4} \times \operatorname{Spin}_{6}$ and $\operatorname{Spin}_{8}$ respectively, and hence consist of branes at fixed angles.

\begin{tabular}{|c|l|}
\hline Codimension $d$ & \multicolumn{1}{|c|}{ Fractions $\nu$} \\
\hline \hline 5 & $\frac{1}{32} \rightarrow \frac{1}{16} \rightarrow \frac{3}{32} \rightarrow \frac{1}{8}$ \\
4 & $\frac{1}{16} \rightarrow \frac{1}{8} \rightarrow \frac{3}{16}$ \\
3 & $\frac{1}{8}$ \\
2 & $\frac{1}{4}$ \\
0 & $\frac{1}{2}$ \\
\hline
\end{tabular}

TABLE 1. Fractions of supersymmetry appearing in configurations of two M5-branes at angles, in terms of the codimension of the intersection. Arrows indicate progressive specialisation.

We will see that with each such solution there is associated a subgroup $G \subset \operatorname{Spin}_{10}$ preserving some spinor, whose maximal torus $\mathbb{T}(G)$ contains the transformations $\widehat{R}(\theta)^{2}$. As discussed above, the fraction $\nu$ is determined from the fact that $32 \nu$ is the number of zero weights of $G$, or equivalently singlets of $\mathbb{T}(G)$, acting on the half-spinor representation $\Delta_{+}$of $\operatorname{Spin}_{10}$. A solution will have an intersection of codimension $d$ whenever $\mathbb{T}(G) \subset \operatorname{Spin}_{2 d} \subset \operatorname{Spin}_{10}$.

3.2. Some regular subgroups of $\operatorname{Spin}_{10}$. It is sufficient to consider only those regular subgroups $G \subset \operatorname{Spin}_{10}$ which leave invariant a spinor. A list of some regular subgroups of $\operatorname{Spin}_{10}$ is given in Figure 1 .

The groups in the Figure are organised in the following fashion. The first row consists of subgroups of $\operatorname{Spin}_{10}$, the second of subgroups of $\operatorname{Spin}_{8}$, the third of $\operatorname{Spin}_{7}$, the fourth of $\operatorname{Spin}_{6}$, and the fifth of $\operatorname{Spin}_{4}$. Actually $\mathrm{SU}_{2} \times \mathrm{SU}_{3}$ and its subgroups $\mathrm{U}_{1} \times \mathrm{SU}_{2} \supset \mathrm{U}_{1}$ are contained in $\operatorname{Spin}_{4} \times \operatorname{Spin}_{6} \subset \operatorname{Spin}_{10}$, whereas $\operatorname{Sp}_{1} \times \operatorname{Sp}_{1}$ and its subgroups $\operatorname{Sp}_{1} \supset \mathrm{U}_{1}$ are contained in $\operatorname{Spin}_{4} \times \operatorname{Spin}_{4} \subset \operatorname{Spin}_{8}$. All the subgroups in the Figure are known to preserve a spinor of $\operatorname{Spin}_{10}$, whence so will their maximal tori. Indeed, as shown for example in [8], the possible isotropy groups of nonzero spinors in $\Delta_{+}$are $\mathrm{SU}_{5}, \mathrm{Spin}_{7}$ and their intersection $\mathrm{SU}_{4}$; and as 


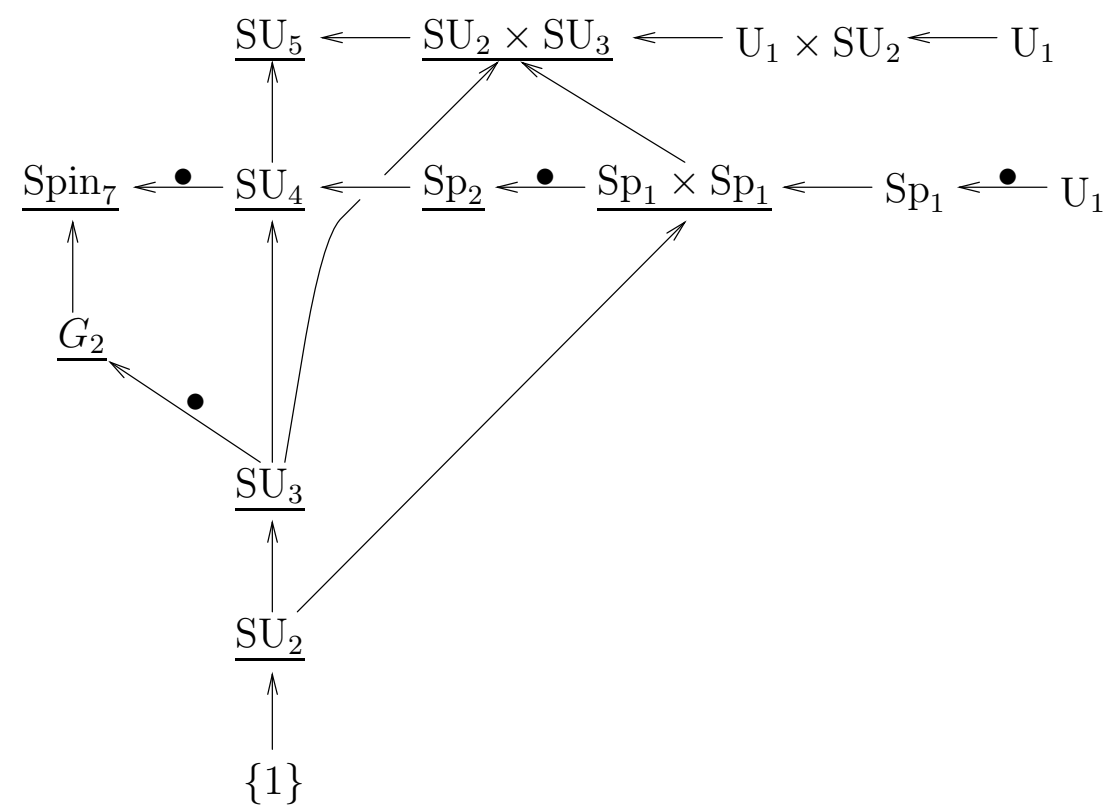

FiguRE 1. Regular subgroups of $\operatorname{Spin}_{10}$ associated with intersecting brane configurations, with every arrow representing an embedding. Embeddings adorned with a $\bullet$ are such that the maximal tori agree. Underlined groups can appear as holonomy groups of spin riemannian manifolds.

we can see by the embedding diagrams all the groups in the Figure are contained in one of these. We therefore expect that the first row should reproduce those fractions in Table 1 corresponding to configurations whose intersection has codimension 5 , the second row should reproduce those of codimension 4, and so on. The last row simply corresponds to the case of coincident branes in which half the supersymmetry is always preserved. Notice that there are more groups in the Figure than fractions in Table 1. This discrepancy can be explained by the fact that some of the groups in Figure 1 1 share the same maximal torus. Since for the case of only two branes the rotation relating them can always be chosen to be in some maximal torus, two groups which share the same maximal tori are indistinguishable in that their two M5-brane configurations will be identical. This happens whenever the embedding relates groups of equal rank. For example, $\mathrm{Spin}_{7}$ and $\mathrm{SU}_{4}$ both have rank 3 and have the same maximal torus in $\mathrm{Spin}_{8}$, and so do $\mathrm{Sp}_{2}$ and $\mathrm{Sp}_{1} \times \mathrm{Sp}_{1}$ which have rank 2, and $\mathrm{Sp}_{1}$ and $\mathrm{U}_{1}$ which have rank 1. Furthermore under the embedding $\operatorname{Spin}_{6} \subset \operatorname{Spin}_{7}$, the maximal tori of $G_{2}$ and $\mathrm{SU}_{3}$ also agree, since the maximal tori of $\mathrm{Spin}_{7}$ and of its $\operatorname{Spin}_{6}$ subgroup are the same. Taking these isomorphisms into account we now see that there as many fractions in Table 1 as there 
are distinct maximal tori in Figure 1. Two features of the Figure are worth remarking. One is the vertical special unitary series $\mathrm{SU}_{5} \supset$ $\mathrm{SU}_{4} \supset \mathrm{SU}_{3} \supset \mathrm{SU}_{2} \supset \mathrm{SU}_{1} \cong\{1\}$. The other is the horizontal spin series in the second row: $\operatorname{Spin}_{7} \supset \operatorname{Spin}_{6} \cong \mathrm{SU}_{4} \supset \operatorname{Spin}_{5} \cong \mathrm{Sp}_{2} \supset$ $\mathrm{Spin}_{4} \cong \mathrm{Sp}_{1} \times \mathrm{Sp}_{1} \supset \mathrm{Spin}_{3} \cong \mathrm{Sp}_{1} \supset \mathrm{Spin}_{2} \cong \mathrm{U}_{1}$. In particular this series suggests that there should be a configuration with the further subgroup $\operatorname{Spin}_{1} \cong \mathbb{Z}_{2}$. We will see how this group arises later on.

3.3. Detailed analysis. We now turn to the case-by-case analysis of this correspondence. We will simply decompose the half-spin representation $\Delta_{+}$of $\operatorname{Spin}_{10}$ into irreducible representations of the relevant group $G$, and simply count the number of zero weights; that is, $\mathbb{T}(G)$ singlets. This yields the fraction $\nu$. The codimension $d$ can be measured by decomposing the vector representation of $\operatorname{Spin}_{10}$ and counting the number of zero weights. If a group $G$ has $k$ zero weights in the vector representation of $\operatorname{Spin}_{10}$, then the corresponding configuration will factor through $\operatorname{Spin}_{10-k}$, whence it will have codimension $d=\left\lfloor\frac{10-k}{2}\right\rfloor$. It is actually possible to reproduce the explicit relations on the angles which were found in 22] by considering the explicit embedding of $G$ in $\operatorname{Spin}_{10}$ and comparing their maximal tori. We will however refrain from doing this here. The results of this section are summarised in Table 2 at the end of the section. Much use has been made of Slansky's Physics Report [24] in reaching some of the results we are about to describe. We will therefore follow tradition and refer to irreducible representations by their dimensions in agreement with [24]. In this notation, the half-spin representation $\Delta_{+}$of $\operatorname{Spin}_{10}$ is denoted $\underline{\mathbf{1 6}}^{*}$, whereas the vector representation is $\underline{\mathbf{1 0}}$. One small notational disagreement worth mentioning concerns the symplectic groups: we call $\mathrm{Sp}_{n}$ what in 24] would be $\mathrm{Sp}_{2 n}$. In our conventions, $\mathrm{Sp}_{1} \cong \mathrm{SU}_{2}$.

3.4. Pointlike intersections. We now describe codimension by codimension the possible groups responsible for the different configurations in 22. We start with codimension $d=5$ which corresponds to branes which intersect at a point.

$\underline{\mathrm{SU}_{5} \subset \operatorname{Spin}_{10}}$. The branching rules associated with this embedding are

$$
\underline{10}=\underline{5} \oplus \underline{5}^{*} \quad \text { and } \quad \underline{16}^{*}=\underline{1} \oplus \underline{5} \oplus \underline{10}^{*} .
$$

Since we are interested in the number of singlets of the maximal torus of $\mathrm{SU}_{5}$, we must count the number of zero weights in the weight space decomposition of the irreducible representations appearing in the branching rules. This can be easily worked out for the representations at hand. The Dynkin labels of these $\mathrm{SU}_{5}$ representations are $\underline{\mathbf{5}}=(1000)$, $\underline{\mathbf{5}}^{*}=(0001)$ and $\underline{\mathbf{1 0}}^{*}=(0010)$, from which the weight decomposition 
can be easily worked out. Working in a Dynkin basis, we find:

$$
\begin{aligned}
(1000) & \rightarrow(-1100) \rightarrow(0-110) \rightarrow(00-11) \rightarrow(000-1) \\
(0001) & \rightarrow(001-1) \rightarrow(01-10) \rightarrow(1-100) \rightarrow(-1000) \\
(0010) & \rightarrow(01-11) \rightarrow(1-101) \oplus(010-1) \rightarrow(-1001) \oplus(1-11-1) \\
& \rightarrow(-101-1) \oplus(10-10) \rightarrow(-11-10) \rightarrow(0-100),
\end{aligned}
$$

whence we can see that the $\underline{\mathbf{1 0}}$ has no zero weights, whence it is a pointlike intersection, and that $\underline{16}^{*}$ has no zero weights other than the singlet. Thus $\nu=\frac{1}{32}$.

$\mathrm{SU}_{2} \times \mathrm{SU}_{3} \subset \mathrm{Spin}_{4} \times \operatorname{Spin}_{6}$. Let us first consider the branching rules associated with the embedding $\operatorname{Spin}_{4} \times \operatorname{Spin}_{6} \subset \operatorname{Spin}_{10}$. Furthermore, since $\operatorname{Spin}_{4} \times \mathrm{Spin}_{6}$ is isomorphic to $\mathrm{SU}_{2} \times \mathrm{SU}_{2} \times \mathrm{SU}_{4}$, we will work with this group instead. The branching rules are

$$
\underline{10}=(\underline{\mathbf{2}}, \underline{\mathbf{2}}, \underline{\mathbf{1}}) \oplus(\underline{\mathbf{1}}, \underline{\mathbf{1}}, \underline{\mathbf{6}}) \quad \text { and } \quad \underline{\mathbf{1 6}}^{*}=\left(\underline{\mathbf{2}}, \underline{\mathbf{1}}, \underline{4}^{*}\right) \oplus(\underline{\mathbf{1}}, \underline{\mathbf{2}}, \underline{4}) \text {. }
$$

Under $\mathrm{SU}_{3} \subset \mathrm{SU}_{4}$ we have the following branching rules:

$$
\underline{\mathbf{6}}=\underline{\mathbf{3}} \oplus \underline{\mathbf{3}}^{*} \quad \underline{\mathbf{4}}=\underline{\mathbf{1}} \oplus \underline{\mathbf{3}} \quad \text { and } \quad \underline{4}^{*}=\underline{\mathbf{1}} \oplus \underline{\mathbf{3}}^{*} .
$$

Of the three possible embeddings $\mathrm{SU}_{2} \subset \mathrm{SU}_{2} \times \mathrm{SU}_{2}$, the diagonal embedding would have a singlet in the $\underline{\mathbf{1 0}}$ since $\underline{\mathbf{2}} \otimes \underline{\mathbf{2}}=\underline{\mathbf{3}} \oplus \underline{\mathbf{1}}$, hence we must embed into the left factor or into the right. Under $\mathrm{SU}_{2}^{L} \times \mathrm{SU}_{3} \subset$ $\operatorname{Spin}_{10}$, we find

$$
\begin{aligned}
\underline{\mathbf{1 0}} & =2(\underline{\mathbf{2}}, \underline{\mathbf{1}}) \oplus(\underline{\mathbf{1}}, \underline{\mathbf{3}}) \oplus\left(\underline{\mathbf{1}}, \underline{\mathbf{3}}^{*}\right) \\
\underline{\mathbf{1 6}}^{*} & =2(\underline{\mathbf{1}}, \underline{\mathbf{1}}) \oplus(\underline{\mathbf{2}}, \underline{\mathbf{1}}) \oplus 2(\underline{\mathbf{1}}, \underline{\mathbf{3}}) \oplus\left(\underline{\mathbf{2}}, \underline{\mathbf{3}}^{*}\right) ;
\end{aligned}
$$

whereas under $\mathrm{SU}_{2}^{R} \times \mathrm{SU}_{3} \subset \operatorname{Spin}_{10}$, we find

$$
\begin{aligned}
\underline{\mathbf{1 0}} & =2(\underline{\mathbf{2}}, \underline{\mathbf{1}}) \oplus(\underline{\mathbf{1}}, \underline{\mathbf{3}}) \oplus\left(\underline{\mathbf{1}}, \underline{\mathbf{3}}^{*}\right) \\
\underline{\mathbf{1 6}}^{*} & =2(\underline{\mathbf{1}}, \underline{\mathbf{1}}) \oplus(\underline{\mathbf{2}}, \underline{\mathbf{1}}) \oplus 2\left(\underline{\mathbf{1}}, \underline{\mathbf{3}}^{*}\right) \oplus(\underline{\mathbf{2}}, \underline{\mathbf{3}}) .
\end{aligned}
$$

It follows that in either of the two cases, the $\underline{\mathbf{1 0}}$ has no zero weights, whereas the $\underline{16}^{*}$ has precisely two, coming from the singlets. In summary, this is a pointlike intersection with $\nu=\frac{1}{16}$.

$\mathrm{U}_{1} \times \mathrm{SU}_{2} \subset \operatorname{Spin}_{4} \times \operatorname{Spin}_{6}$. This group $\mathrm{U}_{1} \times \mathrm{SU}_{2}$ is actually a subgroup of $\mathrm{SU}_{2} \times \mathrm{SU}_{3}$. As discussed above there are two such subgroups of $\operatorname{Spin}_{4} \times \operatorname{Spin}_{6}$ depending on how the $\mathrm{SU}_{2}$ embeds in $\mathrm{Spin}_{4}$. Either of the two cases yields the same results, so we will choose to work with $\mathrm{SU}_{2}^{L} \times \mathrm{SU}_{3}$. There are many conjugacy classes of $\mathrm{U}_{1} \times \mathrm{SU}_{2}$ subgroups of this group, but only one will give rise to a pointlike intersection with $\nu=\frac{3}{32}$. Consider the maximal subgroup $\mathrm{SU}_{2} \times \mathrm{U}_{1}$ of $\mathrm{SU}_{3}$. Under $\mathrm{SU}_{2}^{L} \times \mathrm{SU}_{2} \times \mathrm{U}_{1} \subset \mathrm{SU}_{2}^{L} \times \mathrm{SU}_{3}$, the $\underline{\mathbf{1 0}}$ and $\underline{\mathbf{1 6}}^{*}$ of $\mathrm{Spin}_{10}$ break up as

$$
\begin{aligned}
\underline{\mathbf{1 0}}^{*} & =2(\underline{\mathbf{2}}, \underline{\mathbf{1}})_{0} \oplus(\underline{\mathbf{1}}, \underline{\mathbf{1}})_{-2} \oplus(\underline{\mathbf{1}}, \underline{\mathbf{2}})_{1} \oplus(\underline{\mathbf{1}}, \underline{\mathbf{1}})_{2} \oplus(\underline{\mathbf{1}}, \underline{\mathbf{2}})_{-1} \\
\underline{\mathbf{1 6}}^{*} & =2(\underline{\mathbf{1}}, \underline{\mathbf{1}})_{0} \oplus(\underline{\mathbf{2}}, \underline{\mathbf{1}})_{0} \oplus 2(\underline{\mathbf{1}}, \underline{\mathbf{1}})_{-2} \oplus 2(\underline{\mathbf{1}}, \underline{\mathbf{2}})_{1} \oplus(\underline{\mathbf{2}}, \underline{\mathbf{1}})_{2} \oplus(\underline{\mathbf{2}}, \underline{\mathbf{2}})_{-1} .
\end{aligned}
$$


Because we do not desire any zero weights in the $\underline{\mathbf{1 0}}$, the extra zero weights in the $\underline{16}^{*}$ must come out of representations which do not appear in the $\underline{\mathbf{1 0}}$, namely $(\underline{\mathbf{2}}, \underline{\mathbf{1}})_{2} \oplus(\underline{\mathbf{2}}, \underline{\mathbf{2}})_{-1} \subset \underline{\mathbf{1 6}}^{*}$.

The $\mathrm{U}_{1} \times \mathrm{SU}_{2}$ subgroup of interest is built as follows. The $\mathrm{SU}_{2}$ factor is the same as the $\mathrm{SU}_{2}$ factor in $\mathrm{SU}_{2}^{L} \times \mathrm{SU}_{2} \times \mathrm{U}_{1}$, whereas the $\mathrm{U}_{1}$ factor will be embedded into $\mathrm{U}_{1}^{L} \times U_{1}$ in such a way that, if a representation has weights $(\alpha, \beta)$ relative to $\mathrm{U}_{1}^{L} \times \mathrm{U}_{1}$, it will have weight $2 \alpha+\beta$ relative to the $\mathrm{U}_{1}$ of interest. Therefore under this $\mathrm{U}_{1} \times \mathrm{SU}_{2}$ the branching rules are

$$
\begin{aligned}
\underline{\mathbf{1 0}} & =3 \underline{\mathbf{1}}_{-2} \oplus 3 \underline{\mathbf{1}}_{2} \oplus \underline{\mathbf{2}}_{1} \oplus \underline{\mathbf{2}}_{-1} \\
\underline{\mathbf{1 6}}^{*} & =3 \underline{\mathbf{1}}_{0} \oplus \underline{\mathbf{1}}_{2} \oplus 3 \underline{\mathbf{1}}_{-2} \oplus 3 \underline{\mathbf{2}}_{1} \oplus \underline{\mathbf{1}}_{4} \oplus \underline{\mathbf{2}}_{-3} .
\end{aligned}
$$

It is evident that there are no zero weights in the $\underline{\mathbf{1 0}}$ but there are three in the $\underline{16}^{*}$, whence, as advertised, this is a pointlike intersection with $\nu=\frac{3}{32}$. In order to specialise this configuration further without decreasing the codimension, it is necessary to find a subgroup of $\mathrm{U}_{1} \times \mathrm{SU}_{2}$ which has new singlets in the $\underline{\mathbf{1 6}}^{*}$ but not in the $\underline{\mathbf{1 0}}$. This means that this subgroup must break $\underline{\mathbf{1}}_{4} \oplus \underline{\mathbf{2}}_{-3}$ but not any other other subrepresentations.

$\mathrm{U}_{1} \subset \operatorname{Spin}_{4} \times \operatorname{Spin}_{6}$. This $\mathrm{U}_{1}$ subgroup of the $\mathrm{U}_{1} \times \mathrm{SU}_{2}$ group discussed above is such that the subrepresentation $\underline{\mathbf{2}}_{-3} \subset \underline{\mathbf{1 6}}^{*}$ has a singlet. In other words, under this $\mathrm{U}_{1}$, a representation with weights $(\alpha, \beta)$ under $\mathrm{U}_{1} \times \mathrm{SU}_{2}$ will have weight $3 \alpha+\beta$. We can therefore read off the branching rules from those above:

$$
\begin{aligned}
\underline{\mathbf{1 0}} & =4[-2] \oplus 4[2] \oplus[4] \oplus[-4] \\
\underline{\mathbf{1 6}}^{*} & =4[0] \oplus[2] \oplus 6[-2] \oplus 4[4] \oplus[-6] .
\end{aligned}
$$

This configuration has $d=5$ and $\nu=\frac{1}{8}$. The subgroup $\mathbb{Z}_{6}$ defined as the kernel of the representation $\underline{\mathbf{1}}_{-6}$ can be shown to yield a pointlike intersection with $\nu=\frac{5}{32}$.

3.5. Stringlike intersections. The stringlike intersections are those for which $G$ is a subgroup of $\operatorname{Spin}_{8}$ : two such branes share a common one-dimensional subspace. Under $\operatorname{Spin}_{8} \subset \operatorname{Spin}_{10}$, the branching rules are

$$
\underline{10}=2 \underline{1} \oplus \underline{8}_{\mathrm{v}} \quad \text { and } \quad \underline{16}^{*}=\underline{8}_{\mathrm{s}} \oplus \underline{8}_{\mathrm{c}} .
$$

$\operatorname{Spin}_{7} \subset \operatorname{Spin}_{8}$. There are three conjugacy classes of $\operatorname{Spin}_{7} \operatorname{Subgroups}$ $\mathrm{Spin}_{8}$, two of which leave the vector representation irreducible. They can be distinguished by which one of the half-spin representations they break. In either case, the decomposition of the $\underline{16}^{*}$ of $\operatorname{Spin}_{10}$ is the same, since both $\underline{\boldsymbol{8}}_{\mathrm{s}}$ and $\underline{\boldsymbol{8}}_{\mathrm{c}}$ appear. Indeed, we have the following branchings:

$$
\underline{10}=\underline{8} \oplus 2 \underline{1} \quad \text { and } \quad \underline{16}^{*}=\underline{1} \oplus \underline{\mathbf{7}} \oplus \underline{\mathbf{8}} .
$$


In order to count singlets of the maximal torus, we perform a weight decomposition of these irreducible representations. Their Dynkin labels are $\underline{\boldsymbol{7}}=(100)$ and $\underline{\boldsymbol{8}}=(001)$. Therefore we find the following weights:

$$
\begin{aligned}
(100) & \rightarrow(-110) \rightarrow(0-12) \rightarrow(000) \rightarrow(01-2) \rightarrow(1-10) \rightarrow(-100) \\
(001) & \rightarrow(01-1) \rightarrow(1-11) \rightarrow(-101) \oplus(10-1) \rightarrow(-11-1) \\
& \rightarrow(0-11) \rightarrow(00-1) .
\end{aligned}
$$

Therefore we see that there are two zero weights, coming from the two singlets, in the decomposition of the $\underline{\mathbf{1 0}}$ and also two zero weights, one from the singlet and one from the $\underline{\mathbf{7}}$ in the decomposition of the $\underline{16}^{*}$. In other words, it has $d=4$ and $\nu=\frac{1}{16}$.

$\mathrm{SU}_{4} \subset \mathrm{Spin}_{8}$. The same configuration is also associated with the group $\overline{\mathrm{SU}_{4} \subset \operatorname{Spin}_{7}} \subset \mathrm{Spin}_{8}$, since as we already mentioned, the maximal tori coincide. The branchings in this case are

$$
\underline{\mathbf{1 0}}=2 \underline{\mathbf{1}} \oplus \underline{\mathbf{4}} \oplus \underline{4}^{*} \quad \text { and } \quad \underline{\mathbf{1 6}}^{*}=2 \underline{\mathbf{1}} \oplus \underline{\mathbf{4}} \oplus \underline{4}^{*} \oplus \underline{\mathbf{6}} .
$$

The Dynkin labels of these irreducible representations are $\underline{4}=(100)$, $\underline{\mathbf{4}}^{*}=(001)$ and $\underline{\mathbf{6}}=(010)$; whence the weight decompositions follow:

$$
\begin{aligned}
& (100) \rightarrow(-110) \rightarrow(0-11) \rightarrow(00-1) \\
& (001) \rightarrow(01-1) \rightarrow(1-10) \rightarrow(-100) \\
& (010) \rightarrow(1-11) \rightarrow(-101) \oplus(10-1) \rightarrow(-11-1) \rightarrow(0-10) .
\end{aligned}
$$

Hence all zero weights come from the singlets: two in the $\underline{\mathbf{1 0}}$ and two in the $\underline{16}^{*}$, yielding a stringlike intersection with $\nu=\frac{1}{16}$. In order to specialise further without decreasing the codimension, we need to consider a subgroup $G \subset \mathrm{SU}_{4}$ which has a singlet in the $\underline{\mathbf{6}}$, but none in the $\underline{4}$ or $\underline{4}^{*}$.

$\underline{\mathrm{Sp}_{2} \subset \operatorname{Spin}_{8}}$. The branching rules under $\mathrm{Sp}_{2} \subset \mathrm{SU}_{4}$ are given by

$$
\underline{4}=\underline{4} \quad \underline{4}^{*}=\underline{4} \quad \text { and } \quad \underline{6}=\underline{1} \oplus \underline{5} ;
$$

whence we can write down the decompositions of the $\underline{\mathbf{1 0}}$ and the $\underline{\mathbf{1 6}}^{*}$ under $\operatorname{Sp}_{2} \subset \operatorname{Spin}_{10}$ :

$$
\underline{\mathbf{1 0}}=2 \underline{\mathbf{1}} \oplus 2 \underline{\mathbf{4}} \quad \text { and } \quad \underline{\mathbf{1 6}}^{*}=3 \underline{\mathbf{1}} \oplus 2 \underline{\mathbf{4}} \oplus \underline{\mathbf{5}} .
$$

The Dynkin labels are $\underline{\boldsymbol{4}}=(10)$ and $\underline{\mathbf{5}}=(01)$, and their weights are

$$
\begin{aligned}
& (10) \rightarrow(-11) \rightarrow(1-1) \rightarrow(-10) \\
& (01) \rightarrow(2-1) \rightarrow(00) \rightarrow(-21) \rightarrow(0-1) .
\end{aligned}
$$

Therefore there are exactly two zero weights in the $\underline{\mathbf{1 0}}$, coming from the singlets, and four zero weights in the $\underline{16}^{*}$, three coming from the singlets and one from the $\underline{\mathbf{5}}$. Hence we see that $d=4$ and $\nu=\frac{1}{8}$. To specialise further (and not increase $d$ ) we must get a singlet in the $\underline{\mathbf{5}}$ but none in the $\underline{4}$. 
$\mathrm{Sp}_{1} \times \mathrm{Sp}_{1} \subset \operatorname{Spin}_{4} \times \operatorname{Spin}_{4}$. The same configuration can be obtained with the subgroup $\mathrm{Sp}_{1} \times \mathrm{Sp}_{1} \subset \mathrm{Sp}_{2}$. In terms of $\mathrm{SU}_{2} \times \mathrm{SU}_{2} \times \mathrm{SU}_{2} \times \mathrm{SU}_{2} \cong$ $\operatorname{Spin}_{4} \times \operatorname{Spin}_{4} \subset \operatorname{Spin}_{8}$, we have the following branching rules:

$$
\begin{aligned}
& \underline{8}_{\mathrm{v}}=(\underline{\mathbf{2}}, \underline{\mathbf{2}}, \underline{1}, \underline{\mathbf{1}}) \oplus(\underline{\mathbf{1}}, \underline{\mathbf{1}}, \underline{2}, \underline{2}) \\
& \underline{\mathbf{s}}_{\mathrm{s}}=(\underline{\mathbf{1}}, \underline{\mathbf{2}}, \underline{\mathbf{1}}, \underline{\mathbf{2}}) \oplus(\underline{\mathbf{2}}, \underline{\mathbf{1}}, \underline{\mathbf{2}}, \underline{\mathbf{1}}) \\
& \underline{\mathbf{8}}_{\mathrm{c}}=(\underline{\mathbf{1}}, \underline{\mathbf{2}}, \underline{\mathbf{2}}, \underline{\mathbf{1}}) \oplus(\underline{\mathbf{2}}, \underline{\mathbf{1}}, \underline{\mathbf{1}}, \underline{\mathbf{2}}) .
\end{aligned}
$$

Since each $\mathrm{Sp}_{1}$ factor in $\mathrm{Sp}_{1} \times \mathrm{Sp}_{1}$ belongs to a different $\mathrm{Spin}_{4}$, there are four possible embeddings of $\mathrm{Sp}_{1} \times \mathrm{Sp}_{1}$ in $\operatorname{Spin}_{4} \times \operatorname{Spin}_{4}$ which correspond to stringlike intersections. They all decompose the $\operatorname{Spin}_{10}$ representations in the same way:

$$
\begin{aligned}
\underline{\mathbf{1 0}} & =2(\underline{\mathbf{1}}, \underline{\mathbf{1}}) \oplus 2(\underline{\mathbf{1}}, \underline{\mathbf{2}}) \oplus 2(\underline{\mathbf{2}}, \underline{\mathbf{1}}) \\
\underline{\mathbf{1 6}}^{*} & =4(\underline{\mathbf{1}}, \underline{\mathbf{1}}) \oplus 2(\underline{\mathbf{1}}, \underline{\mathbf{2}}) \oplus 2(\underline{\mathbf{2}}, \underline{\mathbf{1}}) \oplus(\underline{\mathbf{2}}, \underline{\mathbf{2}}) .
\end{aligned}
$$

All zero weights come from singlets, whence there are two in the $\underline{\mathbf{1 0}}$ and four in the $\underline{\mathbf{1 6}}^{*}$. In other words, this too has $d=4$ and $\nu=\frac{1}{8}$. As in the $\mathrm{SU}_{4} \subset \mathrm{Spin}_{7}$ case, one can show that this configuration is precisely the same as from $\mathrm{Sp}_{2}$, since their maximal tori agree in $\mathrm{Spin}_{8}$. Specialising further requires us to get a singlet in the $(\underline{\mathbf{2}}, \underline{\mathbf{2}})$.

$\mathrm{Sp}_{1} \subset \operatorname{Spin}_{4} \times \operatorname{Spin}_{4}$. This $\mathrm{Sp}_{1}$ subgroup sits diagonally in the group

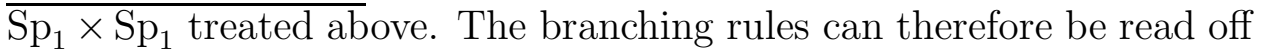
immediately from the ones above:

$$
\underline{\mathbf{1 0}}=2 \underline{\mathbf{1}} \oplus 4 \underline{\mathbf{2}} \quad \text { and } \quad \underline{\mathbf{1 6}}^{*}=5 \underline{\mathbf{1}} \oplus 4 \underline{\mathbf{2}} \oplus \underline{\mathbf{3}} .
$$

Thus there are two zero weights in the $\underline{\mathbf{1 0}}$ coming from the singlets and six zero weights in the $\underline{16}^{*}$, five coming from the singlets and one from the $\underline{\mathbf{3}}$. Hence this has $\overline{d=4}$ and $\nu=\frac{3}{16}$.

$\mathrm{U}_{1} \subset \operatorname{Spin}_{4} \times \operatorname{Spin}_{4}$. The same configuration can be obtained by considering the maximal torus $\mathrm{U}_{1}$ of the above $\mathrm{Sp}_{1}$. Under this subgroup, the branching rules are

$$
\begin{aligned}
\underline{\mathbf{1 0}} & =2[0] \oplus 4[1] \oplus 4[-1] \\
\underline{\mathbf{1 6}}^{*} & =6[0] \oplus[2] \oplus 4[1] \oplus 4[-1] \oplus[-2] ;
\end{aligned}
$$

whence we see that it has $d=4$ and $\nu=\frac{3}{16}$. A further reduction is possible to a subgroup $\mathbb{Z}_{2} \subset \mathrm{U}_{1}$ defined as the kernel of the $[ \pm 2]$ representations. This subgroup still has $d=4$ but now $\nu=\frac{1}{4}$.

3.6. Two-dimensional intersections. Here we have both subgroups of $\operatorname{Spin}_{7}$ and of $\operatorname{Spin}_{6}$. Under $\operatorname{Spin}_{7} \subset \operatorname{Spin}_{10}$ we have

$$
\underline{\mathbf{1 0}}=3 \underline{\mathbf{1}} \oplus \underline{\mathbf{7}} \quad \text { and } \quad \underline{16}^{*}=2 \underline{\mathbf{8}} \text {, }
$$

whereas under $\operatorname{Spin}_{6} \subset \operatorname{Spin}_{10}$ we have

$$
\underline{\mathbf{1 0}}=4 \underline{\mathbf{1}} \oplus \underline{\mathbf{6}} \quad \text { and } \quad \underline{\mathbf{1 6}}^{*}=2 \underline{4} \oplus 2 \underline{4}^{*} .
$$


$G_{2} \subset \operatorname{Spin}_{7}$. Under $G_{2} \subset \operatorname{Spin}_{7}$, the vector representation remains irreducible whereas the spinor has a singlet:

$$
\underline{7}=\underline{7} \quad \text { and } \quad \underline{8}=\underline{1} \oplus \underline{7},
$$

whence we obtain the following branchings for $G_{2} \subset \operatorname{Spin}_{10}$ :

$$
\underline{\mathbf{1 0}}=3 \underline{\mathbf{1}} \oplus \underline{\mathbf{7}} \quad \text { and } \quad \underline{\mathbf{1 6}}^{*}=2 \underline{\mathbf{1}} \oplus 2 \underline{\mathbf{7}} .
$$

The Dynkin label of the $\underline{7}$ is $(01)$, whence its weight decomposition is

$$
(01) \rightarrow(1-1) \rightarrow(-12) \rightarrow(00) \rightarrow(1-2) \rightarrow(-11) \rightarrow(0-1) .
$$

Therefore we see that there are four zero weights in the $\underline{\mathbf{1 0}}$, three from the singlets and one from the $\underline{7}$ and also four zero weights from the $\underline{16}^{*}$, two from the singlets and two from the $\underline{\mathbf{7}}$. This then has $d=3$ and $\nu=\frac{1}{8}$. Notice that this configuration admits no further specialisation with the same codimension. We can however obtain the same configuration with a smaller group.

$\underline{\mathrm{SU}_{3} \subset \operatorname{Spin}_{6}}$. Under $\mathrm{SU}_{3} \subset G_{2}$, we have that

$$
\underline{\mathbf{7}}=\underline{\mathbf{1}} \oplus \underline{\mathbf{3}} \oplus \underline{\mathbf{3}}^{*} \text {. }
$$

Therefore under $\mathrm{SU}_{3} \subset \operatorname{Spin}_{10}$ we find

$$
\underline{\mathbf{1 0}}=4 \underline{\mathbf{1}} \oplus \underline{\mathbf{3}} \oplus \underline{\mathbf{3}}^{*} \quad \text { and } \quad \underline{\mathbf{1 6}}^{*}=4 \underline{\mathbf{1}} \oplus 2 \underline{\mathbf{3}} \oplus 2 \underline{\mathbf{3}}^{*} .
$$

All zero weights come from singlets and we have four in each representation, hence this is also a membrane-like intersection with $\nu=\frac{1}{8}$. In fact, one can show that this is precisely the same configuration as the one from $G_{2}$, since under the embedding $\operatorname{Spin}_{6} \subset \operatorname{Spin}_{7}$, under which the respective maximal tori agree, the maximal tori of $G_{2}$ and $\mathrm{SU}_{3}$ also agree.

3.7. Three-dimensional intersections. Intersections with codimension $d=2$ are such where the rotation leaves three directions invariant, hence the rotation belongs to a $\mathrm{SO}_{4}$ subgroup of $\mathrm{SO}_{10}$ one for which the vector representation contains six singlets. In terms of $\mathrm{SU}_{2} \times \mathrm{SU}_{2} \cong \operatorname{Spin}_{4} \subset \operatorname{Spin}_{10}$, we find the following branching rules:

$$
\underline{\mathbf{1 0}}=6(\underline{\mathbf{1}}, \underline{\mathbf{1}}) \oplus(\underline{\mathbf{2}}, \underline{\mathbf{2}}) \quad \text { and } \quad \underline{\mathbf{1 6}}^{*}=4(\underline{\mathbf{1}}, \underline{\mathbf{2}}) \oplus 4(\underline{\mathbf{2}}, \underline{\mathbf{1}}) .
$$

$\mathrm{SU}_{2} \subset \mathrm{Spin}_{4}$. Again there are two possible embeddings of $\mathrm{SU}_{2}$ in $\mathrm{SU}_{2} \times$

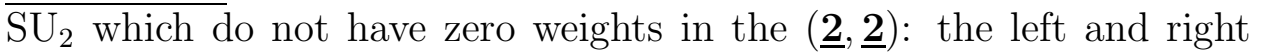
embeddings. Under either one we see that

$$
\underline{\mathbf{1 0}}=6 \underline{\mathbf{1}} \oplus 2 \underline{\mathbf{2}} \quad \text { and } \quad \underline{\mathbf{1 6}}^{*}=8 \underline{\mathbf{1}} \oplus 4 \underline{\mathbf{2}} .
$$

All zero weights come from singlets, of which there are six in the $\underline{\mathbf{1 0}}$ and eight in the $\underline{16}^{*}$. In other words this configuration has $d=2$ and $\nu=\frac{1}{4}$. Again no further specialisation is possible with the same codimension, since the same irreducible representations appear in the decompositions of the $\underline{\mathbf{1 0}}$ and $\underline{\mathbf{1 6}} *$. 


\begin{tabular}{|c|c|c|c|c|c|c|c|}
\hline \multirow{2}{*}{$\begin{array}{c}\text { Codimension } \\
d\end{array}$} & \multirow{2}{*}{$\begin{array}{c}\text { Group } \\
\quad G\end{array}$} & \multirow{2}{*}{$\begin{array}{c}\text { Rank } \\
\quad \ell\end{array}$} & \multicolumn{2}{|c|}{ Singlets } & \multicolumn{2}{|c|}{$\begin{array}{c}\text { Zero } \\
\text { Weights }\end{array}$} & \multirow{2}{*}{$\begin{array}{c}\text { Fraction } \\
\nu \\
\end{array}$} \\
\hline & & & 10 & $\underline{16}^{*}$ & 10 & $\underline{16}^{*}$ & \\
\hline \multirow{4}{*}{5} & $\mathrm{SU}_{5}$ & 4 & 0 & 1 & 0 & 1 & $\frac{1}{32}$ \\
\hline & $\mathrm{SU}_{2} \times \mathrm{SU}_{3}$ & 3 & 0 & 2 & 0 & 2 & $\frac{1}{16}$ \\
\hline & $\mathrm{U}_{1} \times \mathrm{SU}_{2}$ & 2 & 0 & 3 & 0 & 3 & $\frac{3}{32}$ \\
\hline & $\mathrm{U}_{1}$ & 1 & 0 & 4 & 0 & 4 & $\frac{1}{8}$ \\
\hline \multirow{6}{*}{4} & $\operatorname{Spin}_{7}$ & 3 & 2 & 1 & 2 & 2 & $\frac{1}{16}^{\star}$ \\
\hline & $\mathrm{SU}_{4}$ & 3 & 2 & 2 & 2 & 2 & $\frac{1}{16}$ \\
\hline & $\mathrm{Sp}_{2}$ & 2 & 2 & 3 & 2 & 4 & $\frac{1}{8}^{\star}$ \\
\hline & $\mathrm{Sp}_{1} \times \mathrm{Sp}_{1}$ & 2 & 2 & 4 & 2 & 4 & $\frac{1}{8}$ \\
\hline & $\mathrm{Sp}_{1}$ & 1 & 2 & 5 & 2 & 6 & $\frac{3}{16}^{\star}$ \\
\hline & $\mathrm{U}_{1}$ & 1 & 2 & 6 & 2 & 6 & $\frac{3}{16}$ \\
\hline \multirow[t]{2}{*}{3} & $G_{2}$ & 2 & 3 & 2 & 4 & 4 & $\frac{1}{8}^{\star}$ \\
\hline & $\mathrm{SU}_{3}$ & 2 & 4 & 4 & 4 & 4 & $\frac{1}{8}$ \\
\hline 2 & $\mathrm{SU}_{2}$ & 1 & 6 & 8 & 6 & 8 & $\frac{1}{4}$ \\
\hline 0 & $\{1\}$ & 0 & 10 & 16 & 10 & 16 & $\frac{1}{2}$ \\
\hline
\end{tabular}

TABLE 2. Singlets and zero-weights in the decompositions of the $\underline{10}$ and $\underline{16}^{*}$ of $\operatorname{Spin}_{10}$ under the different groups in Figure 1, and fraction of supersymmetry in the resulting configuration. Fractions $\nu$ where $32 \nu$ is not equal to the number of singlets in the $\underline{\mathbf{1 6}}^{*}$ have been starred.

3.8. Summary of results. In summary, to every supersymmetric configuration of two M5-branes, we have assigned a subgroup $G$ of $\operatorname{Spin}_{10}$ in such a way that the preserved supersymmetry corresponds to the number of invariant spinors under the action of (the maximal torus of) $G$. These results are summarised in Table 2, which contains the subgroups of $\operatorname{Spin}_{10}$ discussed above, not including the finite subgroups. We list the rank of the group as well as the number of singlets in the vector and spinor representations, and the number of singlets of the maximal torus, that is the zero weights. The fraction of the supersymmetry which is preserved can be read off from the number of zero weights in the spinor representation, and the codimension can be read off from the number of zero weights in the vector representation. In 
some cases the fraction does not agree with the singlets of the group, which means that the maximal torus leaves more spinors invariant than the group itself. In these cases there is a smaller subgroup of $\operatorname{Spin}_{10}$ which shares the maximal torus.

These results do not just provide a group-theoretical backbone to the results in [22], but provide a basis for the extension of these results to configurations involving more than two branes, to which we now turn.

\section{Multiple intersections}

In this section we turn our attention to the case of multiply intersecting branes. The general case of more than two intersecting branes is not immediately amenable to the kind of analysis we have been discussing above. The difficulty arises already for three intersecting branes. Suppose the three branes are parallel to start with and rotate one of them away by a rotation $R_{1}$ and a second one by a rotation $R_{2}$. Unless $R_{1}$ and $R_{2}$ commute, they will not belong to the same maximal torus, and hence it will be impossible to choose a basis so that the matrices representing $R_{1}$ and $R_{2}$ will have the general form (3). In other words, we will not be able to work only with maximal tori. A different approach is therefore needed. In this section we will set up the problem, review what is known and show that one can also associate an "automorphism" group with a given supersymmetric configuration, in such a way that the codimension and the fraction of supersymmetry preserved can be computed in terms of that group. Strictly speaking we prove a theorem which determines a lower bound for the fraction $\nu$ in terms of group theory, and we conjecture, based on a growing body of evidence, that the bound is actually saturated. We will also comment on how with every such group one can associate a geometry in the sense of Harvey \& Lawson and we will mention some examples of such geometries.

4.1. Statement of the problem. The problem is to characterise the supersymmetric configurations of $m$ intersecting M5-branes and determine the fraction $\nu$ of the supersymmetry which is preserved. At a mathematical level, this problem can be formulated as follows. Let $\Delta$ be a fixed irreducible representation of the Clifford algebra $C \ell_{1,10}$. It is a real 32-dimensional representation which remains irreducible under $\operatorname{Spin}_{10,1} \subset \mathrm{C} \ell_{1,10}$. Let $\xi$ be a 5 -plane in $\bigwedge^{5} \mathbb{E}^{10}$, and let $e_{0} \wedge \xi$ denote the tangent plane to the worldvolume of an M5-brane. Let us define the following subspace of $\Delta$ :

$$
\Delta(\xi) \equiv\left\{\varepsilon \in \Delta \mid\left(e_{0} \wedge \xi\right) \cdot \varepsilon=\varepsilon\right\} .
$$

$\Delta(\xi) \subset \Delta$ is a real 16-dimensional subspace. If $\eta$ is another 5-plane, then define

$$
\Delta(\xi \cup \eta) \equiv \Delta(\xi) \cap \Delta(\eta)
$$


and so on. Let $\xi_{1} \equiv \xi, \xi_{2}, \ldots, \xi_{m}$ be $m 5$-planes. We say that the configuration $\cup_{i=1}^{m} \xi_{i}$ is supersymmetric if and only if

$$
\Delta\left(\cup_{i=1}^{m} \xi_{i}\right)=\bigcap_{i=1}^{m} \Delta\left(\xi_{i}\right) \neq\{0\} .
$$

A supersymmetric configuration $\cup_{i=1}^{m} \xi_{i}$ is said to preserve a fraction $\nu$ of the supersymmetry, where

$$
32 \nu=\operatorname{dim} \Delta\left(\cup_{i=1}^{m} \xi_{i}\right) .
$$

Clearly $\nu$ can only take the values $\frac{1}{32}, \frac{1}{16}, \frac{3}{32}, \ldots, \frac{1}{2}$. Two questions are fundamental.

Question 1. How can one characterise the supersymmetric configurations $\cup_{i=1}^{m} \xi_{i}$ ?

Question 2. What fraction $\nu$ of the supersymmetry is preserved by a given supersymmetric configuration $\cup_{i=1}^{m} \xi_{i}$ ?

Both questions have been answered in [22] for $m=2$. In [1] (see also [15]) we answered the first question for arbitrary $m$ using techniques of calibrated geometry, a result we will presently recall, since it will be the starting point of our analysis. After doing that we will present a partial answer to the second question for arbitrary $m$.

4.2. Supersymmetric configurations of $G$-related planes. How about the second question for $m>2$ ? In this section we will present a partial answer to this question and will conjecture a complete answer based on computer experimentation; but first let us briefly recall the result of [1] concerning the first question for arbitrary $m$. See also the recent work of $15 \|$.

If a configuration $\cup_{i=1}^{m} \xi_{i}$ is supersymmetric, then there is at least one nonzero spinor $\varepsilon \in \Delta$ which belongs to $\Delta\left(\xi_{i}\right)$ for all $i$. As shown in [1], this implies that $\xi_{i}$ are calibrated by a 5 -form which can be constructed from $\varepsilon$. The nature of the form depends on the isotropy subgroup of the spinor. Nonzero spinors in eleven dimensions have one of two possible isotropy subgroups: $\mathrm{SU}_{5} \subset \operatorname{Spin}_{10}$ and $\operatorname{Spin}_{7} \ltimes \mathbb{R}^{9}$ 《8. In the former case, the 5 -form is special lagrangian, and the planes $\xi_{i}$ are special lagrangian planes. Because the special lagrangian grassmannian is isomorphic to $\mathrm{SU}_{5} / \mathrm{SO}_{5}$, we see that the planes are all related to each other by $\mathrm{SU}_{5}$ transformations. On the other hand, the latter group intersects $\operatorname{Spin}_{10}$ in an $\operatorname{Spin}_{7}$ subgroup, and one can show that the 5form is now of the form $v^{*} \wedge \Omega$, where $v \in \mathbb{E}^{10}$ is a fixed vector, $v^{*}$ is the dual 1-form annihilating $v^{\perp}$ and such that $\left\langle v^{*}, v\right\rangle=1$, and $\Omega$ is a Cayley form on an $\mathbb{E}^{8} \subset v^{\perp}$. This means that each plane $\xi_{i}$ is of the form $v \wedge \zeta_{i}$, where $\zeta_{i}$ are Cayley planes. Because the Cayley grassmannian is acted on transitively by $\operatorname{Spin}_{7}$, we see that the $\zeta_{i}$, and hence the $\xi_{i}$, are related to each other by $\operatorname{Spin}_{7}$ transformations. These 
5 -planes all intersect at least in the subspace spanned by $v$, whence these configurations have codimension $d \leq 4$. The generic fraction $\nu$ is $\frac{1}{32}$ in either case, unless $m=2$ in which case, as we have seen, planes related by $\operatorname{Spin}_{7}$ transformations are actually related by $\mathrm{SU}_{4}$ transformations, and the fraction doubles.

Notice that $\mathrm{SU}_{5}$ and $\mathrm{Spin}_{7}$ contain all other subgroups in Figure 1 . Therefore it is is conceivable that demanding that the $\xi_{i}$ be related by transformations in some group $G$, where $G \subset \mathrm{SU}_{5}$ or $G \subset \operatorname{Spin}_{7}$ (or both, in which case $G \subset \mathrm{SU}_{4}$ ), one should obtain configurations with possibly lower codimension and a higher fraction of supersymmetry. The codimension $d$ is given by $d=\left\lfloor\frac{10-k}{2}\right\rfloor$, where $k$ is the number of $G$-singlets in the vector representation $\underline{\mathbf{1 0}}$ of $\operatorname{Spin}_{10}$. One would expect that the fraction $\nu$ of the supersymmetry would be similarly given by $\frac{1}{32} \times$ the number of singlets in the $\underline{\mathbf{1 6}}^{*}$. Hence the degeneracies corresponding to the starred fractions in Table 2 would be lifted. We have already seen that this is true for $\mathrm{Spin}_{7}$, which now yields a fraction $\nu=\frac{1}{32}$. Similarly we expect that $\mathrm{Sp}_{2}$ should yield a fraction $\nu=\frac{3}{32}$, $\operatorname{Sp}_{1} \subset \operatorname{Spin}_{8}$ a fraction $\nu=\frac{5}{32}$ and $G_{2}$ a fraction $\nu=\frac{1}{16}$. We have so far been unable to prove this, but we can prove that the fraction is at least that. We do this now, but first a definition.

Let $G\left(5, \mathbb{E}^{10}\right)$ denote the grassmannian of oriented 5-planes in $\mathbb{E}^{10}$. It is acted on transitively by $\mathrm{SO}_{10}$ with isotropy $\mathrm{SO}_{5} \times \mathrm{SO}_{5}$. A given subgroup $G \subset \operatorname{Spin}_{10}$ acts on $G\left(5, \mathbb{E}^{10}\right)$ by restricting the action of $\mathrm{SO}_{10}$ to the subgroup to which $G$ gets mapped under the canonical covering map $\operatorname{Spin}_{10} \rightarrow \mathrm{SO}_{10}$. We can therefore consider the decomposition of the grassmannian into $G$-orbits.

Definition 1. Let $\left\{\xi_{i}\right\}$ be $m$ oriented 5-planes in $\mathbb{E}^{10}$. We say that they are $G$-related, if they all lie in the same $G$-orbit and furthermore $G$ is the smallest such subgroup of $\operatorname{Spin}_{10}$.

The results of [回] can be rephrased as saying that a configuration $\cup_{i=1}^{m} \xi_{i}$ is supersymmetric if and only the planes are $G$-related, where $G \subset \operatorname{Spin}_{7}$ or $G \subset \mathrm{SU}_{5}$ or both so that $G \subset \mathrm{SU}_{4}$. Because both $\operatorname{Spin}_{7}$ and $\mathrm{SU}_{5}$ preserve a spinor, so will $G$. Let $\Delta^{G} \subset \Delta$ denote the subspace of $G$-invariant spinors in $\Delta$. Let the fraction $\nu_{G}$ be defined by

$$
\nu_{G} \equiv \frac{1}{64} \operatorname{dim} \Delta^{G} .
$$

Equivalently, $32 \nu_{G}$ is the number of linearly independent $G$-singlets in the $\underline{\mathbf{1 6}}^{*}$ (or the $\underline{\mathbf{1 6}}$ ) of $\operatorname{Spin}_{10}$, which can be read off from Table 2 for the subgroups discussed in Section 3. We are now ready to prove the following result.

Theorem 1. Let $\cup_{i=1}^{m} \xi_{i}$ be a supersymmetric configuration of oriented 5 -planes in $\mathbb{E}^{10}$ which are $G$-related. Then the fraction $\nu$ of the supersymmetry which is preserved obeys $\nu \geq \nu_{G}$. 
Proof. Let $\xi \equiv \xi_{1}$, say, be one of the planes, and let $\pi=e_{0} \wedge \xi$. Also let $\pi_{i}=e_{0} \wedge \xi_{i}$, for $i=1, \ldots, m$, so that $\pi_{1}=\pi$. Because the $\xi_{i}$ are $G$ related, and $G \subset \operatorname{Spin}_{10}$ acts trivially on $e_{0}$, so are the $\pi_{i}$. This means that there are group elements $g_{i} \in G$, unique modulo the isotropy of $\xi$, so that $\xi_{i}=g_{i} \xi$ and $\pi_{i}=g_{i} \pi$. Now let $\varepsilon \in \Delta^{G} \cap \Delta(\xi)$; that is, $\varepsilon$ is a $G$-invariant spinor which obeys $\pi \cdot \varepsilon=\varepsilon$. It is plain that $\varepsilon$ also obeys $\pi_{i} \cdot \varepsilon=\varepsilon$ for all $i$. Indeed,

$$
\begin{array}{rlrl}
\pi_{i} \cdot \varepsilon & =g_{i} \cdot \pi \cdot g_{i}^{-1} \cdot \varepsilon & \\
& =g_{i} \cdot \pi \cdot \varepsilon & \\
& =g_{i} \cdot \varepsilon & \left(\varepsilon \in \Delta^{G}\right) \\
& =\varepsilon, & (\varepsilon \in \Delta(\xi)) \\
& & & \left(\varepsilon \in \Delta^{G}\right)
\end{array}
$$

whence $\varepsilon \in \Delta\left(\xi_{i}\right)$ for all $i$. In other words, we have shown that

$$
\Delta^{G} \cap \Delta(\xi) \subset \Delta\left(\cup_{i=1}^{m} \xi_{i}\right),
$$

whence

$$
\nu \equiv \frac{1}{32} \operatorname{dim} \Delta\left(\cup_{i=1}^{m} \xi_{i}\right) \geq \frac{1}{32} \operatorname{dim}\left(\Delta^{G} \cap \Delta(\xi)\right) .
$$

We will now show that $\Delta^{G} \cap \Delta(\xi)$ has half the dimension of $\Delta^{G}$.

Because $\pi \cdot \pi=\mathbb{1}$, we have a decomposition

$$
\Delta=\Delta^{+} \oplus \Delta^{-}
$$

into eigenspaces of $\pi$. Clearly $\Delta^{+}=\Delta(\xi)$. The above decomposition allows us to decompose $\Delta^{G}$ :

$$
\Delta^{G}=\Delta_{+}^{G} \oplus \Delta_{-}^{G}
$$

where $\Delta_{ \pm}^{G}=\Delta^{G} \cap \Delta^{ \pm}$. Now consider the action of $e_{0}$ on $\Delta$. Because $G \subset \operatorname{Spin}_{10}, g \cdot e_{0}=e_{0} \cdot g$ for all $g \in G$. Therefore $e_{0}$ preserves $\Delta^{G}$. Furthermore, $e_{0} \cdot \pi=-\pi \cdot e_{0}$, whence $e_{0}$ maps $\Delta^{+}$to $\Delta^{-}$, and also $\Delta_{+}^{G}$ to $\Delta_{-}^{G}$. Because $e_{0} \cdot e_{0}=-\mathbb{1}$, it is an isomorphism, and $\Delta_{ \pm}^{G}$ have the same dimension: one half the dimension of $\Delta^{G}$. In other words, $\Delta^{G} \cap \Delta(\xi)$ has half the dimension of $\Delta^{G}$. Together with (11), the theorem follows.

There is a large body of evidence which suggests that the inequality in the Theorem is actually saturated. The $m=2$ results described in Section 3 support this, and so do the results of computer experimentation. We therefore feel confident in the validity of the following conjecture. It essentially asserts that there is no accidental supersymmetry, beyond that which is guaranteed by the group theory.

Conjecture 1. A supersymmetric configuration $\cup_{i=1}^{m} \xi_{i}$ of oriented 5planes in $\mathbb{E}^{10}$ which are $G$-related preserves a fraction $\nu_{G}$ of the supersymmetry, where $\nu_{G}$ is given by (10). 
We can give many examples of configurations of an arbitrary number of intersecting M5-branes preserving a certain fraction of the supersymmetry. We simply choose the planes to be $G$-related where $G \subset \operatorname{Spin}_{10}$ is a given subgroup of $\mathrm{SU}_{5}$ or $\operatorname{Spin}_{7}$ (or both). Modulo the conjecture, the fraction $\nu=\nu_{G}$ can then be read off from Table 2, and in any case the fraction will be at least $\nu_{G}$. The possible fractions are as in the $m=2$ case: $\frac{1}{32}, \frac{1}{16}, \frac{3}{32}, \frac{1}{8}, \frac{5}{32}, \frac{3}{16}, \frac{1}{4}$ and $\frac{1}{2}$. These results are summarised in Figure 2. It is worth remarking, however, that for $m=2$, the fraction $\nu=\frac{5}{32}$ appears for branes which are $\Gamma$-related, where $\Gamma$ is a certain $\mathbb{Z}_{6}$ subgroup of $\operatorname{Spin}_{4} \times \operatorname{Spin}_{6} \subset \operatorname{Spin}_{10}$. Using this group, configurations with $\nu=\frac{5}{32}$ would of necessity consist of a finite number of non-coincident branes. In contrast, for generic $m$, this fraction is associated to an $\mathrm{Sp}_{1}$ subgroup of $\operatorname{Spin}_{4} \times \operatorname{Spin}_{4} \subset \operatorname{Spin}_{8}$, whence configurations with $\nu=\frac{5}{32}$ consisting an arbitrary number of non-coincident branes are possible.

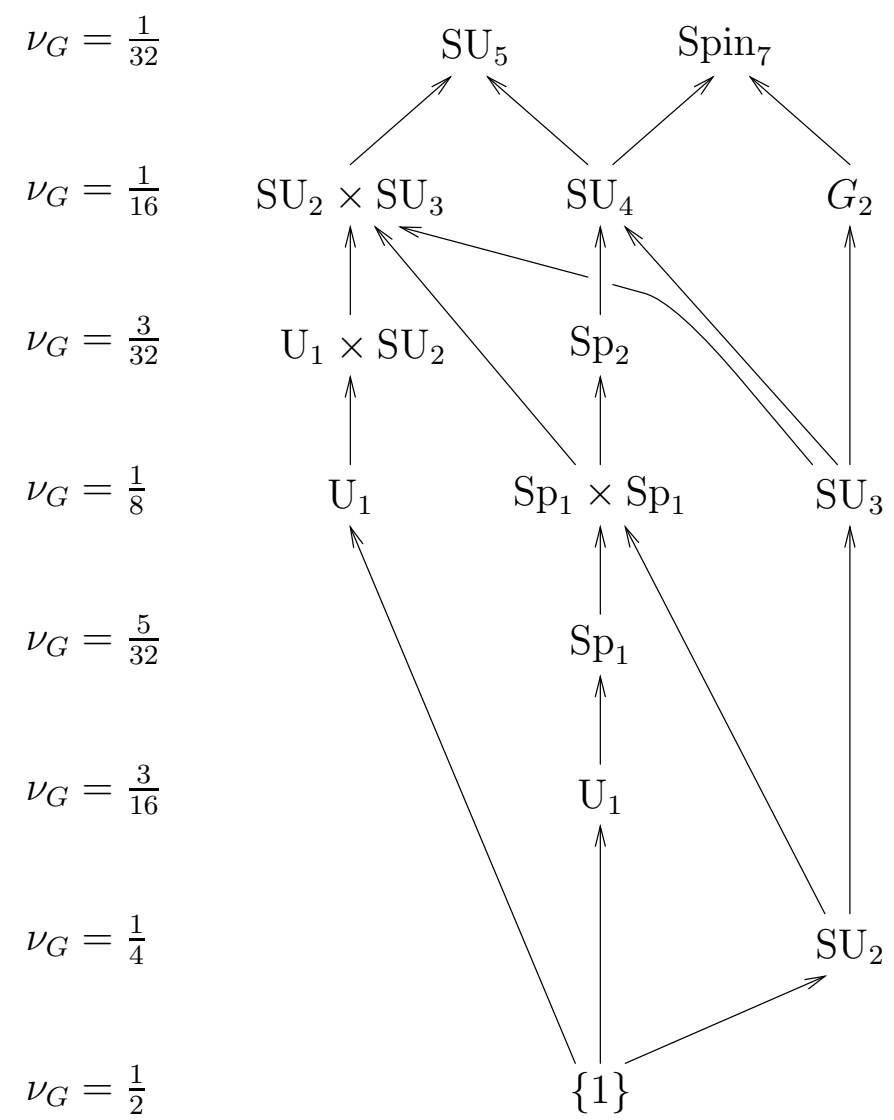

FiguRE 2. Fractions of supersymmetry associated to $G$ related planes as a function of $G$. Each arrow denotes an embedding. 
4.3. Geometry of intersecting brane configurations. We have seen how with a given supersymmetric static configuration of intersecting branes one can associate a Lie subgroup $G \subset \operatorname{Spin}_{10}$ such that the different branes are $G$-related. Moreover we have conjectured a precise relation between the fraction of the supersymmetry preserved by such a configuration and the dimension of the space of $G$-invariant spinors. We will now refine this correspondence and associate with every such configuration a given geometry: this correspondence is most clearly seen in the formalism of calibrated geometry [16]. For a review of the basic notions of calibrated geometry in the present context, see our previous paper [1] as well as references therein. Other recent papers which discuss calibrated geometry in the context of intersecting branes are 15, 14]. In particular 15] contains a complementary treatment of some of the material in this section.

Let $G \subset \operatorname{Spin}_{10}$ and suppose we are given a configuration of $G$ related planes. As proven above, such a configuration preserves at least a fraction $\nu_{G}$ of the supersymmetry. This means that there are at least $32 \nu_{G}$ spinors $\varepsilon_{i}$ which obey

$$
\left(e_{0} \wedge \xi\right) \cdot \varepsilon_{i}=\varepsilon_{i}
$$

for every oriented 5-plane $\xi$ in the configuration. As shown in [1], this means that every such 5 -plane $\xi$ is calibrated by a (constant coefficient) 5 -form $\Omega_{i}$ in $\mathbb{E}^{10}$ which can be obtained from $\varepsilon_{i}$ by squaring. Every such form $\Omega_{i}$ defines a face of the grassmannian of oriented 5planes in $\mathbb{E}^{10}$, known as the $\Omega_{i}$-grassmannian. By the Theorem, the subset of $G$-related planes containing the given configuration is itself contained in (and conjecturally agrees with) the intersection of the $\Omega_{i^{-}}$ grassmannians. As we will see in many examples below, this subset often turns out to be itself isomorphic to the $\Omega$-grassmannian for some $(p \leq 5)$-form $\Omega$. Therefore it defines a geometry in the sense of Harvey \& Lawson [16].

As explained for example in [21], a $p$-submanifold (with possible selfintersections) of $\mathbb{E}^{10}$ whose tangent spaces lie in the same $\Omega$-grassmannian, is homologically volume-minimising. In other words, the geometry associated with the $\Omega$-grassmannian corresponds to the geometry of minimal $p$-dimensional immersions in some euclidean space $\mathbb{E}^{D \leq 10}$. Given $G \subset \operatorname{Spin}_{10}$, one determines $D$ and $p$ as follows: $10-D$ is the dimension of the $G$-invariant subspace $V^{G}$ of $\mathbb{E}^{10}$, whereas $5-p$ is the dimension of subspace $V^{G} \cap \xi$. Equivalently, $p$ is equal to the codimension $d$ of the configuration. We have not classified all possible $G$, but we have managed to construct a number of examples, which are summarised in Table 3. This table refines Table 2 in that conjugate subgroups of $\operatorname{Spin}_{10}$, while preserving the same fraction of the supersymmetry, can give rise to different geometries. 


\begin{tabular}{|c|c|c|c|}
\hline $\begin{array}{l}\text { Codim. } \\
\quad d\end{array}$ & $\begin{array}{c}\text { Group } \\
\text { G }\end{array}$ & $\begin{array}{l}\text { Isotropy } \\
\qquad K \\
\end{array}$ & $\begin{array}{l}\text { Geometry } \\
G / K\end{array}$ \\
\hline 5 & $\begin{array}{c}\mathrm{SU}_{5} \\
\mathrm{SU}_{2} \times \mathrm{SU}_{3}\end{array}$ & $\begin{array}{c}\mathrm{SO}_{5} \\
\mathrm{SO}_{2} \times \mathrm{SO}_{3}\end{array}$ & $\begin{array}{c}\mathrm{SLAG}_{5} \\
\left(\mathbb{C}_{1} \text { or } \mathrm{SLAG}_{2}\right) \times \mathrm{SLAG}_{3}\end{array}$ \\
\hline 4 & $\begin{array}{c}\mathrm{Spin}_{7} \\
\mathrm{SU}_{4} \\
\mathrm{SU}_{4} \\
\mathrm{Sp}_{2} \\
\mathrm{Sp}_{2} \\
\mathrm{Sp}_{1} \times \mathrm{Sp}_{1} \\
\mathrm{Sp}_{1} \times \mathrm{Sp}_{1} \\
\mathrm{Sp}_{1} \\
\mathrm{U}_{1}\end{array}$ & $\begin{array}{c}\left(\mathrm{SU}_{2}\right)^{3} / \mathbb{Z}_{2} \\
\mathrm{SO}_{4} \\
\mathrm{~S}\left(\mathrm{U}_{2} \times \mathrm{U}_{2}\right) \\
\mathrm{U}_{2} \\
\mathrm{Sp}_{1} \times \mathrm{Sp}_{1} \\
\mathrm{U}_{1} \times \mathrm{U}_{1} \\
\mathrm{Sp}_{1} \\
\mathrm{U}_{1} \\
\{1\}\end{array}$ & $\begin{array}{c}\text { Cayley } \\
\text { SLAG }_{4} \\
\mathbb{C}_{2} \\
\mathbb{C L A G}_{2} \\
\mathrm{H}_{1} \\
\mathbb{C}_{1} \times \mathbb{C}_{1} \\
(3,1) \text { in } \mathbb{1 1} \\
(3,2) \text { in } \mathbb{1 1} \\
(3,3) \text { in } \mathbb{1 1}\end{array}$ \\
\hline 3 & $\begin{array}{c}G_{2} \\
\mathrm{SU}_{3}\end{array}$ & $\begin{array}{l}\mathrm{SO}_{4} \\
\mathrm{SO}_{3}\end{array}$ & $\begin{array}{l}\text { Associative } \\
\text { SLAG }_{3}\end{array}$ \\
\hline 2 & $\begin{array}{l}\mathrm{SU}_{3} \\
\mathrm{SU}_{2}\end{array}$ & $\begin{array}{c}\mathrm{S}\left(\mathrm{U}_{2} \times \mathrm{U}_{1}\right) \\
\mathrm{SO}_{2}\end{array}$ & $\begin{array}{c}\mathbb{C}_{1} \\
\mathbb{C}_{1} \text { or } \mathrm{SLAG}_{2}\end{array}$ \\
\hline
\end{tabular}

TABLE 3. Geometries associated with intersecting brane configurations. Listed are some groups $G$ leaving spinors invariant, and the isotropy subgroup $K \subset G$ which leaves the 5-plane $\xi$ invariant. The geometry of the resulting grassmannian $G / K$ is also listed.

Let us comment briefly on these results. These examples have been arrived at by choosing a convenient reference 5-plane $\xi$ and picking a number of linearly independent spinors in $\Delta(\xi)$. The intersection $G$ of their isotropy subgroups inside $\operatorname{Spin}_{10} \subset \mathrm{C} \ell_{1,10}$ can be computed. From this it is a simple matter to determine the intersection $K$ of $G$ with the isotropy subgroup of $\xi$. Many of the calculations have been performed infinitesimally (i.e., using their Lie algebras) using Mathematica. .

We have included in the table only those groups $G$ for which we could determine the geometry. In particular, some groups in Table 2 associated with configurations with $d=5$ are missing. This reflects the present knowledge about the faces of the grassmannian of oriented

\footnotetext{
${ }^{1}$ Details of the calculations can be obtained by email from the authors. They will be made public via our web pages at a later date.
} 
5 -planes in $\mathbb{E}^{10}$. The determination of the faces of the grassmannian $G\left(p, \mathbb{E}^{D}\right)$ of oriented $p$-planes in $\mathbb{E}^{D}$ is not an easy problem whenever $p$ is different from $1,2, D-2$, or $D-1$. To this day, only the cases $(p, D)=(3,6)[10,17,20]$ and $(3,7)$ [18, 20] have been fully solved, whereas there are some partial results for $(p, 8)$ [11].

Some of the geometries in the table are reasonably well-known: the geometries of $p$-dimensional complex $\left(\mathbb{C}_{p}\right)$ or quaternionic $\left(\mathbb{H}_{p}\right)$ submanifolds are classical. The special lagrangian $\left(\mathrm{SLAG}_{p}\right)$, Cayley and associative geometries were discovered by Harvey and Lawson in their foundational essay [16], and have been discussed recently in the context of intersecting branes in [15, 14, 1]. Less known perhaps are the complex lagrangian $(\mathbb{C L A G})$ geometry of $p$-dimensional complex submanifolds in $\mathbb{C}^{2 p}$ which are lagrangian relative to a complex symplectic form, and the geometries of types $(3,1),(3,2)$ and $(3,3)$. These geometries are associated to faces of the grassmannian $G\left(4, \mathbb{E}^{8}\right)$ of oriented 4 -planes in $\mathbb{E}^{8}$ which are calibrated by self-dual 4 -forms. They are discussed, together with explicit representative calibrations, in [11].

Finally we should mention that there are more faces in the grassmannian than the ones discussed here: we have only discussed those faces which contain tangent spaces to supersymmetric brane configurations. The other faces correspond to cycles which are not supersymmetric, yet are still minimal. It may be interesting to study these faces in more detail, particularly in the context of Kaluza-Klein supergravity duality.

\section{Conclusions And OPEn PROBlems}

In this paper we have outlined a complete characterisation of configurations of multiply intersecting branes at angles in terms of subgroups of $\operatorname{Spin}_{10}$ preserving some spinors. We believe that this framework might be useful in the algebraic approach to intersecting branes and in principle reduces the classification of such configurations to a problem in group theory, which is roughly speaking the decomposition of the grassmannian of oriented 5-planes in ten dimensions in terms of orbits of subgroups $G$ of $\operatorname{Spin}_{10}$ contained in the isotropy of some spinor. This approach suggests some open problems.

The obvious open problem is settling the Conjecture, but there are other problems as well. One should do a systematic search of subgroups of $\mathrm{SU}_{5}$ and $\operatorname{Spin}_{7}$ (i.e., of subgroups of $\operatorname{Spin}_{10}$ which are contained in the isotropy of a spinor) and determine the fraction $\nu_{G}$ for them; maybe one finds fractions which are not listed here. We are not aware of any completeness result. The groups and fractions discussed in this paper are only complete for the case of two intersecting branes [22]. Other groups, maybe even finite groups, may appear when one considers more than two branes. Each such group determines a 'geometry' in the sense 
of [16]. In other words, the orbit under this group of the original M5brane defines a subset of the grassmannian of oriented planes which, as explained at the end of the previous section, can be associated with a certain geometry. It would be interesting to classify these geometries. This is a refinement of the (unsolved) problem of determining the faces of the grassmannian of oriented 5 -planes in $\mathbb{E}^{10}$, since one need only consider those faces which are intersections of the faces exposed by calibrations which can be obtained by squaring spinors.

Another obvious problem, which will be addressed in Part II is to lift the restriction on the types of transformations one is allowed to do on the branes. We have followed [22] and allowed the branes to be merely rotated relative to each other; but in fact, one should allow for general eleven-dimensional Lorentz transformations. A similar analysis is possible and one can classify all the supersymmetric configurations involving only two branes as well as prove some partial results for the case of an arbitrary number of branes [2]. No new fractions seem to emerge in this case either.

There are other interesting aspects of intersecting M-branes which we have not addressed in this paper and for which this approach may be fruitful. The duality between intersecting branes and Kaluza-Klein supergravity [12] should be studied further, as are the supergravity solutions corresponding to these more general configurations. We hope to report on these problems in the future.

\section{ACKNOWLEDGEMENTS}

It is a pleasure to thank Robert Bryant for his helpful correspondence and for sending us his unpublished notes [8], and Sonia Stanciu for useful discussions. In addition, JMF would like to thank Takashi Kimura and the Department of Mathematics of Boston University for the hospitality during part of the time it took to write this paper. BSA is supported by a PPARC Postdoctoral Fellowship, JMF by an EPSRC PDRA and BS by an EPSRC Advanced Fellowship, and we would like to extend our thanks to the relevant research councils for their support.

\section{REFERENCES}

[1] BS Acharya, JM Figueroa-O'Farrill, and B Spence, Branes at angles and calibrated geometry, JHEP 04 (1998) 012, hep-th/9803260.

[2] BS Acharya, JM Figueroa-O'Farrill, B Spence, and S Stanciu, Planes, branes and automorphisms: II. Branes in motion, hep-th/9805176.

[3] V Balasubramanian, F Larsen, and RG Leigh, Branes at angles and black holes, hep-th/9704143.

[4] K Behrndt and M Cvetič, BPS-saturated bound states and tilted p-branes in type ii string theory, Phys. Rev. D56 (1997), 1188.

[5] E Bergshoeff, M de Roo, E Eyras, B Janssen, and JP van der Schaar, Multiple intersections of D-branes and M-branes, hep-th/9612095. 
[6] M Berkooz, MR Douglas, and RG Leigh, Branes intersecting at angles, Nucl. Phys. B480 (1996), 265-278, hep-th/9606139.

[7] JC Breckenridge, G Michaud, and RC Myers, New angles on branes, hep-th/9703041.

[8] RL Bryant, Remarks on spinors in low dimension, Unpublished notes, 1998.

[9] MS Costa and M Cvetič, Non-threshold D-brane bound states and black holes with non-zero entropy, Phys. Rev. D56 (1997), 4834-4843.

[10] J Dadok and FR Harvey, Calibrations on $\mathbb{R}^{6}$, Duke Math. J. 50 (1983), 12311243.

[11] J Dadok, FR Harvey, and F Morgan, Calibrations on $\mathbb{R}^{8}$, Trans. Am. Math. Soc. 307 (1988), 1-40.

[12] JP Gauntlett, GW Gibbons, G Papadopoulos, and PK Townsend, Hyperkähler manifolds and multiply intersecting branes, hep-th/9702202.

[13] JP Gauntlett, DA Kastor, and J Traschen, Overlapping branes in M-theory, Nucl. Phys. B478 (1996), 544.

[14] JP Gauntlett, ND Lambert, and PC West, Branes and calibrated geometries, hep-th/9803216.

[15] GW Gibbons and G Papadopoulos, Calibrations and intersecting branes, hep-th/9803163.

[16] FR Harvey and HB Lawson, Calibrated geometries, Acta Math. 148 (1982), $47-157$.

[17] FR Harvey and F Morgan, The comass ball in $\bigwedge^{3}\left(\mathbb{R}^{6}\right)^{*}$, Ind. U. Math. J. 35 (1986), 145-156.

[18] FR Harvey and F Morgan, The faces of the Grassmannian of three-planes in $\mathbb{R}^{7}$ (Calibrated geometries in $\mathbb{R}^{7}$ ), Invent. math. 83 (1986), 191-228.

[19] MM Sheikh Jabbari, Classification of different branes at angles, hep-th/9710121.

[20] F Morgan, The exterior algebra $\bigwedge^{k} \mathbb{R}^{n}$ and area minimization, Linear Algebra Appl. 66 (1985), 1-28.

[21] F Morgan, Area-minimizing surfaces, faces of grassmannians, and calibrations, Am. Math. Monthly 95 (1988), 813-822.

[22] N Ohta and PK Townsend, Supersymmetry of M-branes at angles, Phys. Lett. B418 (1998), 77, hep-th/9710129.

[23] G Papadopoulos and PK Townsend, Intersecting M-branes, Phys. Lett. 380B (1996), 273.

[24] R Slansky, Group theory for unified model building, Phys. Rep. 79 (1981), no. $1,1-128$.

[25] PK Townsend, M-branes at angles, hep-th/9708074.

[26] AA Tseytlin, Harmonic superposition of M-branes, Nucl. Phys. B475 (1996), 273.

Department of Physics

Queen Mary and Westfield College

Mile End RoAd

LONDON E1 4NS, UK

E-mail address: r.acharya@qmw.ac.uk

E-mail address: j.m.figueroa@qmw.ac.uk

E-mail address: b.spence@qmw.ac.uk 